\title{
Topological theory of magnetism in nanostructured ferromagnets
}

\author{
Arno Holz \\ Fachrichtung Theoretische Physik, Universität des Saarlandes, 66041 Saarbrücken, Germany \\ Claudio Scherer \\ Instituto de Fisica, Universidade Federal do Rio Grande do Sul, 90.000 Porto Alegre, Rio Grande do Sul, Brazil \\ (Received 7 September 1993; revised manuscript received 11 March 1994)

\begin{abstract}
It is postulated that in nanostructured ferromagnets, e.g., $\mathrm{Ni}, \mathrm{Fe}$, and $\mathrm{Co}$, exchange and magnetostatic energy dominate crystalline anisotropy energy and therefore the usual domain structure imposed via anisotropy is replaced by a structure consisting of topological defects. The defects are nonlinear solutions of the classical Heisenberg Hamiltonian of spins modified by magnetostatic energy, have the topological properties of disclinations, and are partly classified by the Hopf index. Coercive force is a consequence of entanglements of disclinations, pinning, and mutual obstruction during crossing processes correlated to local (spin) conductivity. Domain boundaries at surfaces are replaced by topological point defects of opposite topological charge generated pairwise.
\end{abstract}

\section{INTRODUCTION}

Magnetism in nanostructured (NS) ferromagnets, e.g., $\mathrm{Fe}, \mathrm{Co}$, and $\mathrm{Ni}$, differs considerably from that observed in crystalline and polycrystalline materials as has been pointed out by Gleiter, ${ }^{1}$ and Kisker, $^{2}$ and Schaefer et $a l .{ }^{3}$ First, no domain boundaries ${ }^{4}$ at surfaces are observed using the Bitter technique and magneto-optic Kerr effect. $^{1-3}$ Second, coercive field strength $H_{c}$ in NS ferromagnets, e.g., $\mathrm{Ni}$, is much larger than in polycrystalline samples, and its temperature dependence also differs. ${ }^{2,3}$ On the other hand, the temperature dependence of the low-temperature saturation magnetization $M_{S}$ in NS Ni decreases with a $T^{2}$ law upon increasing the temperature similarly as in crystalline samples. ${ }^{2,3}$ This is in contrast with the usual $T^{3 / 2}$ law observed in ferromagnets with localized integer valued (in multiples of Bohr's magneton $\mu_{B}$ ) magnetic moments. Third, there is an indication ${ }^{2,3}$ that in NS Ni magnetism disorders in two steps, separated by $\sim 85 \mathrm{~K}$, yielding a reduction of magnetization of $\sim 20 \%$ in the first step, and being attributed to the boundary component of the sample. Because parallel to these processes grain growth in this temperature domain. sets in, a theoretical interpretation of that property is less straightforward. For a review of the ordinary magnetic properties of $\mathrm{Fe}, \mathrm{Co}$, and $\mathrm{Ni}$ we refer to Wohlfahrt. ${ }^{5}$

In the following we consider this problem from the perspective of microscopic magnetism postulating that exchange and magnetostatic energy dominate crystalline anisotropy energy. The theory is based on an idea of Kronmüller ${ }^{6}$ suggesting that microscopic magnetic structures are characterized by at least three characteristic "exchange lengths"

$$
\begin{aligned}
& \kappa_{S}^{-1}=\left(J / 2 \pi M_{S}^{2}\right)^{1 / 2}, \\
& \kappa_{K}^{-1}=(J / K)^{1 / 2}, \\
& \kappa_{H}^{-1}=\left(2 J / H M_{S}\right)^{1 / 2},
\end{aligned}
$$

where $J, M_{S}, K$, and $H$ are the exchange constant, saturation magnetization, crystal anisotropy, and applied magnetic field, respectively. The three characteristic lengths refer to the magnetostatic energy originating from the stray fields (1), the crystalline anisotropy (2), and the applied magnetic field (3). Magnetoelastic effects are usually two orders of magnitude smaller, i.e., their characteristic exchange length $\kappa_{M}^{-1}=\left(J / \lambda\left|\sigma_{i}\right|\right)^{1 / 2}\left(\lambda\right.$ and $\sigma_{i}$ are constant of magnetostriction and internal stress, respectively) is much larger than the three characteristic lengths introduced above, but in plastically deformed and work hardened material $\kappa_{M}^{-1}$ diminishes and then it also plays a significant role as has been pointed out by Träuble. ${ }^{7}$ Because no work hardening is observed in NS metals, ${ }^{1}$ it will be ignored in the following. The characteristic length scales indicated above are naturally dominated by the magnetic correlation length $\xi$ defined over the twopoint magnetic correlation function; $\xi$ obeys the condition $\xi \ll \kappa_{\sigma}^{-1}$ for $\sigma=S, K, H, M$ up to temperatures pretty close to the Curie temperature $T_{C}$.

The physical significance of the length scales (1), (2), and (3) originates from the fact that any change of the magnetization structure via activation of one of its corresponding energies takes place over the respective length unit, where the proportionality factor depends on the boundary conditions. In case more than one type of energy is activated in a magnetic configuration constrained by boundary conditions, its local structure is determined by the shortest characteristic length. In the case of $\mathrm{Fe}$ and $\mathrm{Ni}^{6}$ we have, e.g., at $H=1000 \mathrm{Oe}$ and room temperature, $\kappa_{S}^{-1} \cong 3.3 \mathrm{~nm}, \kappa_{K}^{-1} \cong 20.9 \mathrm{~nm}$, and $\kappa_{H}^{-1} \cong 15.3 \mathrm{~nm}$, and $\kappa_{S}^{-1} \cong 7.6 \mathrm{~nm}, \kappa_{K}^{-1} \cong 45.3 \mathrm{~nm}$, and $\kappa_{H}^{-1} \cong 18.9 \mathrm{~nm}$, respectively. Observe also that the width of a Bloch wall, which is naturally determined by $\kappa_{K}^{-1}$, is of the order of $10 \kappa_{K}^{-1}$, whereas the width of a Néel wall is of the order of $\pi \kappa_{S}^{-1}$.

Nanostructured material, of the type schematically illustrated in Fig. 1, consists of a compacted and sintered 


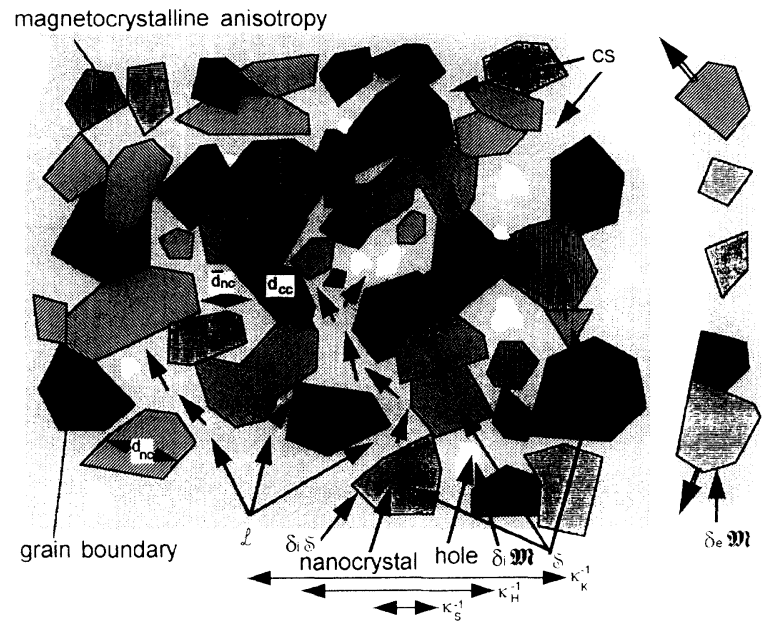

FIG. 1. Schematic illustration of a two-dimensional cross section of NS material characterized by three characteristic geometric lengths, $d_{\mathrm{NC}}, \overline{d_{\mathrm{NC}}}$, and $d_{\mathrm{CC}}$, and three characteristic magnetic lengths, $\kappa_{K}^{-1}, \kappa_{H}^{-1}$, and $\kappa_{S}^{-1}$. $\mathcal{S}$ (skeleton) and $\mathcal{L}$ (labyrinth) represent the global structural units of our model of NS material, with $d_{\mathrm{NC}}$ and $\overline{d_{\mathrm{NC}}}$ the linear scales of $\mathcal{S}$ and $\mathcal{L}$, respectively. $d_{\mathrm{CC}}$ represents the linear scale of crystallographic correlations of $\mathcal{S}$. $\partial_{i} \mathcal{M}$, and $\partial_{e} \mathcal{M}$ represent internal and external boundaries of the sample $\mathcal{M}$ with $\partial_{i} \mathcal{M}, \partial_{e} \mathcal{M}$ and $\partial_{i} \rho$ representing the boundaries of holes (pores), the surface of the sample, and the surface of $\mathcal{S}$, respectively.

aggregate of nanometer-sized crystals. Kisker ${ }^{2}$ and Schaefer et al. ${ }^{3}$ studied the magnetic properties of such materials formed from $\mathrm{Ni}$ and $\mathrm{Fe}$ with crystal sizes $\left(d_{\mathrm{NC}}\right)$ of the order of $\sim 10-12 \mathrm{~nm}$ for $\mathrm{Ni}$ and $\sim 16-19 \mathrm{~nm}$ for Fe. Suppose that the nanometer-sized crystals in NS material form a skeleton like structure with a short-range crystallographic correlation $\left(d_{\mathrm{CC}}\right)$. Furthermore, the labyrinthine domain, whose boundary is the skeleton, is filled with material of unknown structure [called complex structured (CS)] and partly empty (holes and pores). The skeleton $(\mathscr{S})$ and labyrinth $(\mathcal{L})$ are roughly complementary to each other in $M$, i.e., $\mathcal{S}+\mathcal{L}=\mathcal{M}$, where $\mathcal{M}$ is the domain in $\mathbb{R}^{3}$ occupied by the sample. Due to the existence of wedge- and star-shaped boundary domains in $\mathcal{f}$ it is hard to imagine that the boundary conditions imposed by the shape of $\mathcal{S}$ favor any of the known uniform microscopic structures of matter (amorphous, liquid, or gas). As a matter of fact the density distribution of CS material should be $\gtrsim 50 \%$ of bulk matter, ${ }^{1}$ depending on how much it fills up the labyrinthine domain.

As length scale for the labyrinth we use $\bar{d}_{\mathrm{NC}}$, which may be computed over the packing density and an appropriate crystal size correlation function and for NS material satisfies $\bar{d}_{\mathrm{NC}} \ll d_{\mathrm{NC}}$. Note that in Fig. 1 we distinguish between grain boundaries, i.e., the small- or largeangle grain boundaries between crystalline contacts forming the joints of $\mathcal{S}$ and the CS material in $\mathcal{L}$. Furthermore the nanocrystals forming $\mathcal{S}$ may show a characteristic defect structure produced during cluster formation and subsequent compaction and may be subject to internal strain balanced at the joints of $\mathcal{S}$, and at the boun- daries with $\mathcal{L} \cdot d_{\mathrm{NC}}, \bar{d}_{\mathrm{NC}}$, and $d_{\mathrm{CC}}$ are three additional length scales in the system, with the ordering $\bar{d}_{\mathrm{NC}} \ll d_{\mathrm{NC}} \lesssim d_{\mathrm{CC}}$, not present in amorphous ferromagnets. In addition the latter systems may show a domain structure due to magnetocrystalline anisotropy. For a review of the properties of amorphous ferromagnets we refer to Luborsky. ${ }^{8}$ Note that Schäfer, Hubert, and Herzer ${ }^{9}$ produced magnetic "NS material" by controlled crystallization from a glass alloy and observe domain patterns resembling those of metallic glasses. This shows that the microstructure of NS materials depends strongly on their mode of production, and it is emphasized that the theory developed here refers to compacted and sintered aggregates of nanocrystals.

Observe that a NS material is not a scaled down version of a polycrystalline (PC) material due to the low density of NS material ( $\lesssim 90 \%$ of PC material ${ }^{1}$ ). This implies that the fictitious process of replacing micrometersized crystals in a PC material by nanometer-sized crystals does not lead to the state of matter characteristic of NS material. As a matter of fact, it is possible to postulate models of NS material, where all matter is incorporated in the skeleton and where the labyrinth consists only of holes and pores. These models, however, are not general enough because they imply the existence of a large spectrum of small- and large-angle grain boundaries of nanometer-sized scale, which is not proven. Furthermore, such models do not take account of the modified boundary conditions, to which matter is subject, that is "evaporated" into the labyrinth or deposited there via plastic flow during the process of formation (via compaction and sintering of the material).

It is the purpose of this work to develop a qualitative scenario for magnetic behavior in NS ferromagnets and put it into relation with other modern systems presently studied, e.g., quenched ferrofluids ${ }^{10}$ or ideal Heisenberg magnets. The main idea underlying this paper is based on the observation that due to $\kappa_{K}^{-1} \gg d_{\mathrm{NC}} \gg \kappa_{S}^{-1}$ and the plausible assumption that crystallographic correlations in $\mathcal{S}$ are of the order of $d_{\mathrm{NC}}$, crystal anisotropy energy plays no role in NS ferromagnets. Because the usual domain structure of a ferromagnet is a natural device to reduce magnetostatic energy via elimination of magnetic surface charges one may postulate it to occur also in a NS ferromagnet. Suppose, therefore, that we take such a fictitious domain structure as initial state and release the constraints (torques) imposed by the crystal anisotropy by means of a stochastic reorientation of nanocrystals in $\mathcal{S}$, allowing the system to evolve into a final state. This implies an increase of anisotropy energy $\left(\delta H_{K}\right)$ of the order of $K V / 2$ ( $V$ the volume of the system), which is independent of the subsequent change of magnetization configuration accompanied by a modification of exchange $\left(\delta \mathscr{H}_{J}\right)$ and magnetostatic $\left(\delta \mathscr{H}_{S}\right)$ energy. Instability of the initial and stability of the final state require $0>\delta \mathscr{H}_{J}+\delta \mathscr{H}_{S}>-\delta \mathscr{H}_{K}$, respectively. If the inequality signs are replaced by equality signs the first one would imply stability of a Bloch state without crystal anisotropy and the second one stability of a non-Bloch state in the presence of anisotropy, which is impossible. In case the initial configuration contained only Bloch walls we will 
have $\delta \mathscr{H}_{J}<0$ and $\delta \mathscr{H}_{S}>0$. This implies that the rotation of magnetization $(\nabla \times \mathbf{M})$ contained in the Bloch walls must spread over the system generating a magnetic charge density $(\boldsymbol{\nabla} \cdot \mathbf{M})$. Naturally the same applies if initially Néel walls existed, except that now magnetic charge is distributed over the system.

The classification of final states will be done by topological methods, where two-dimensional (2D) domain walls are replaced by topological defects and where the more important will be called (magnetic) disclinations. The latter roughly may be described by their core geometry and therefore form 1D structures. The linear dimension of the core is $\sim \pi \kappa_{S}^{-1}$. This estimate is based on the assumption that the magnetic charge $\boldsymbol{\nabla} \cdot \mathbf{M}$ is essentially located in the core of disclinations, whereas the different disclinations of a global configuration are connected by field lines with $\boldsymbol{\nabla} \times \mathbf{M} \neq 0$. Because $\mathbf{M}(\mathbf{r})$ is essentially a unit-vector field this assumption has only qualitative significance. It is obvious that in generic cases a mapping between initial and final magnetic configurations (as has been indicated above) will be too complex to be of any practical use, and therefore domains in NS ferromagnets play no role. This applies also to the approach of $T_{C}$, where the topological defects indicated above are generated thermally.

The rest of the paper is organized in six sections. In Sec. II the topological concepts applying to Heisenberg magnets being of interest in micromagnetics are reviewed. Section III is devoted to a qualitative description of quasistatic change of topology in Heisenberg magnets. Such a theory is necessary if one wants to describe transitions between different topological sectors of the configuration space of the system in connection with the coercive force $H_{c}$. It cannot be done within a continuum theory and requires additional parameters. The latter may be derived from a microscopic theory of tunneling processes and essentially are governed by a characteristic time $\eta_{t}$, which has the significance of a short-wavelength $\left(\sim \kappa_{S}^{-1}\right)$ and high-frequency $\left(\sim k_{B} T_{C} / \hbar\right)$ spin resistivity. For $\eta_{t} \rightarrow 0$, topological obstruction cannot be overcome, whereas for $\eta_{t} \rightarrow \infty$ it vanishes. The latter situation occurs upon approaching $T_{C}$. In Sec. IV, low-energy magnetostatic modes are discussed. It is expected that such modes have increased significance in NS ferromagnets due to the necessary presence of magnetic charge at the boundaries between $\mathcal{f}$ and $\mathcal{L}$ in the bulk. In Sec. V, five micromagnetic applications are presented among them the computation of magnetic energy for configurations bounded by planes. Section VI is devoted to a qualitative analysis of the coercive force $H_{c}$ as observed in Refs. 2 and 3, within the theory developed in Secs. III and IV. In Sec. VII some conclusions are drawn.

\section{TOPOLOGICAL CONCEPTS IN HEISENBERG MAGNETS}

Suppose that no crystallographic correlation exists between neighboring crystals of the skeleton, i.e., $d_{\mathrm{NC}}=d_{\mathrm{CC}}$. Then for

$$
\begin{aligned}
& \kappa_{S}^{-1} \ll \kappa_{K}^{-1}, d_{\mathrm{NC}} \ll \kappa_{K}^{-1}, \\
& \pi \kappa_{S}^{-1} \gg \bar{d}_{\mathrm{NC}}, \text { and } \pi \kappa_{S}^{-1} \sim O\left(d_{\mathrm{NC}}\right),
\end{aligned}
$$

crystals will be magnetically "monodomain" and exchange and stray field energies will not allow an optimization of the crystalline anisotropy energy. As a matter of fact, configurational changes of the magnetization should occur in such a way that the exchange energy together with the stray field energy is minimized under the given boundary conditions, due to $\pi \kappa_{S}^{-1} \sim O\left(d_{\mathrm{NC}}\right)$. Since $d_{\mathrm{NC}} \ll \kappa_{K}^{-1}$ and $\pi \kappa_{S}^{-1} \sim O\left(d_{\mathrm{NC}}\right)$, configurational changes cannot take place over Bloch or Néel wall motion, which are the most effective degrees of freedom to yield a small coercive force $H_{c}$ in micrometer-sized PC samples. Under such constraints the magnetization structure of a sample is essentially determined through the external and internal (magnetic) surface structure of $\mathcal{M}$ and the topological defects imposed onto the magnetization.

Note that in PC samples the corresponding characteristic linear lengths $\left(d_{\mathrm{PC}}, \bar{d}_{\mathrm{PC}}\right)$ satisfy $d_{\mathrm{PC}} \sim 10^{2} d_{\mathrm{NC}}$, $\bar{d}_{\mathrm{PC}} \sim \bar{d}_{\mathrm{NC}}$, implying $d_{\mathrm{PC}} \gg \kappa_{K}^{-1}$, and $\bar{d}_{\mathrm{PC}} \ll \kappa_{S}^{-1}$. Due to the constraint imposed by the fixed orientation of grain boundaries, a change of magnetization there will occur over domain walls being a mixture of Bloch and Néel types. Because the latter yields a magnetic charging of grain boundaries, a reduction of magnetostatic energy can be reached over the formation of a magnetic domain structure within grains or along grain boundaries. Configurational change of magnetization in such a system is mainly discussed in terms of the motion of Bloch and Neel walls, but it is obvious that the topological defects introduced below will also be present in such configurations imposing constraints on their effective number of degrees of freedom.

\section{A. Micromagnetics of NS ferromagnets}

Under the conditions stated above, a classical microscopic Hamiltonian to study the time-independent aspects of this problem in the long-wavelength limit is the following: ${ }^{6}$

$$
\mathscr{H}=\mathscr{H}_{S}+\mathscr{H}_{J}+\mathscr{H}_{H}
$$

where $\mathscr{H}_{S}$ refers to the stray field energy,

$$
\begin{gathered}
\mathscr{H}_{S}=\frac{1}{8 \pi} \int_{\mathbf{R}^{3}} d^{3} r \mathbf{H}_{S}^{2}=\frac{1}{2} \int_{\mathcal{M}} d^{3} r\left[\nabla_{r} \cdot \mathbf{M}(\mathbf{r})\right] \Phi_{M}(\mathbf{r}) \\
=-\frac{1}{2} \int_{M} d^{3} r \mathbf{M} \cdot \mathbf{H}_{S}, \\
\Phi_{M}(\mathbf{r})=-\int_{M} \frac{\nabla_{r^{\prime}} \cdot \mathbf{M}\left(\mathbf{r}^{\prime}\right) d^{3} r^{\prime}}{\left|\mathbf{r}-\mathbf{r}^{\prime}\right|}+\int_{\partial^{\prime} M} \frac{\mathbf{M}\left(\mathbf{r}^{\prime}\right) \cdot d^{2} r^{\prime}}{\left|\mathbf{r}-\mathbf{r}^{\prime}\right|} .
\end{gathered}
$$

Here $\Phi_{M}(\mathbf{r})$ and $\mathbf{H}_{S}$ are scalar (magnetic) potential and stray field strength, respectively; $\mathscr{H}_{J}$ refers to the exchange energy

$$
\mathscr{H}_{J}=\int_{\mathcal{M}} d^{3} r J(\mathbf{r})(\nabla \mathbf{N})^{2},
$$

and $\mathscr{H}_{H}$ refers to the energy of the sample in an applied magnetic field $\mathbf{H}$ 


$$
\mathscr{H}_{H}=-\int_{\mathcal{M}} d^{3} r \mathbf{M}(\mathbf{r}) \cdot \mathbf{H}
$$

In (5), (6), and (7) we have introduced for the magnetization density (order parameter field) the notation $\mathbf{M}(\mathbf{r})=M_{S} \mathbf{N}(\mathbf{r})$, so that $\mathbf{N}(\mathbf{r})$ is essentially a unit vector field $\left[O(3)\right.$ order parameter] and $M_{S}=M_{S}(\mathbf{r}, T)$ (scalar order parameter) is the saturation magnetization. $\mathcal{M}$ represents the manifold formed by the NS material, whose internal and external boundaries are denoted by $\partial_{i} \mathcal{M}$ and $\partial_{e} \mathcal{M}$, respectively and $\partial^{\prime} \mathcal{M}=\partial_{i} \mathcal{M}+\partial_{e} \mathcal{M}$ $+\partial_{i} \mathcal{S}+\partial_{i} \mathcal{L}$, with $\partial_{i} \mathcal{} \mathcal{}=\partial_{i} \mathcal{L}$, but opposite orientation. The volume densities $J(r)$ and $M_{S}(r, T)$ may be taken as position dependent, and enter (1), (2), and (3).

In order to provide an interpretation of magnetic properties as a function of temperature, we will also have to compute the magnetic excitational modes of NS material over a time-dependent change of the field $\mathbf{M}(\mathbf{r}, t)$. For that purpose (4) must be supplemented by two timedependent Hamiltonians

$$
\mathscr{H}_{E}=(1 / 8 \pi) \int_{\mathbf{R}^{3}} d^{3} r \mathbf{E} \cdot \mathbf{D}
$$

and $\mathscr{H}_{\dot{S}}$ derived from the Lagrangian

$$
\begin{aligned}
\mathcal{L}_{\dot{S}}=(1 / \gamma) \int_{\mathcal{M}} d^{3} r M_{S}(r, T) \\
\times\left(N_{1} \frac{d N_{2}}{d t}-N_{2} \frac{d N_{1}}{d t}\right) /\left(1-N_{3}\right) .
\end{aligned}
$$

Here $\mathbf{E}$ and $\mathbf{D}$ are electric field strength and displacement field, respectively, and $\gamma=g e / 2 m c$ ( $g$ is the gyromagnetic ratio) and $\mathbf{N}(\mathbf{r}, t)=\left(N_{1}, N_{2}, N_{3}\right)$. $\mathscr{H}_{\dot{s}}$ governs the precessional motion of spin, which may be computed from

$$
\frac{d}{d t} \mathbf{M}(\mathbf{r}, t)=\{\mathbf{M}(\mathbf{r}, t), \mathscr{H}\}_{\mathrm{PB}},
$$

where the Poisson bracket (PB) is evaluated via the formula

$$
\left\{M_{i}(r, t), M_{j}\left(r^{\prime}, t\right)\right\}_{\mathbf{P B}}=\gamma \epsilon_{i j k} \delta\left(\mathbf{r}-\mathbf{r}^{\prime}\right) \mathbf{M}_{k}(\mathbf{r}, t)
$$

and $\epsilon_{i j k}$ is the totally antisymmetric symbol. The timedependent change of electromagnetic fields may be computed from Maxwell's equation in a slow motion approximation.

$$
\begin{aligned}
\left\{\Delta-\frac{4 \pi}{c^{2}} \sigma \partial_{t}-\frac{\epsilon_{0}}{c^{2}} \partial_{t}^{2}\right\} & {\left[\begin{array}{l}
\mathbf{E} \\
\mathbf{B}
\end{array}\right] } \\
& =\left\{\begin{array}{l}
\frac{4 \pi}{c} \partial_{t}(\nabla \times \mathbf{M}) \\
-4 \pi \nabla(\nabla \cdot \mathbf{M})+4 \pi \Delta \mathbf{M} .
\end{array}\right.
\end{aligned}
$$

Here the dielectric constant $\epsilon$ enters in the usual form $\epsilon=\epsilon_{0}-4 \pi i \sigma / \omega$, where also $\epsilon(\mathbf{k}, \omega)$ may be used with the replacements $\mathbf{k} \rightarrow-i \nabla, \omega \rightarrow i \partial_{t}$, in the arguments of $\epsilon_{0}$ and $\sigma$.

Consider first the case where there exist no internal boundaries $\left(\partial_{i} M\right)$ and the densities $J$ and $M_{S}$ are independent of position. This is in any case an idealization but roughly applies to the case that grain boundaries and CS material are magnetic, i.e., the densities $J$ and $M_{S}$ do not diminish in these regions so much, that the skeleton effectively consists of magnetically decoupled nanocrystals interacting only via the long-range stray fields. In that case the external boundary $\left(\partial_{e} \mathcal{M}\right)$ of the system determines the magnetization structure for $\kappa_{S}^{-1}$ and $\kappa_{H}^{-1}>0$ and the system displays ideal Heisenberg magnetism. Suppose that $\kappa_{S}^{-1} \ll<\kappa_{H}^{-1}$, i.e., $\kappa_{S}^{-1}$ is small enough so that the surface will be demagnetized. For the case $(f)$ illustrated in Fig. 2(a) one obtains for instance with
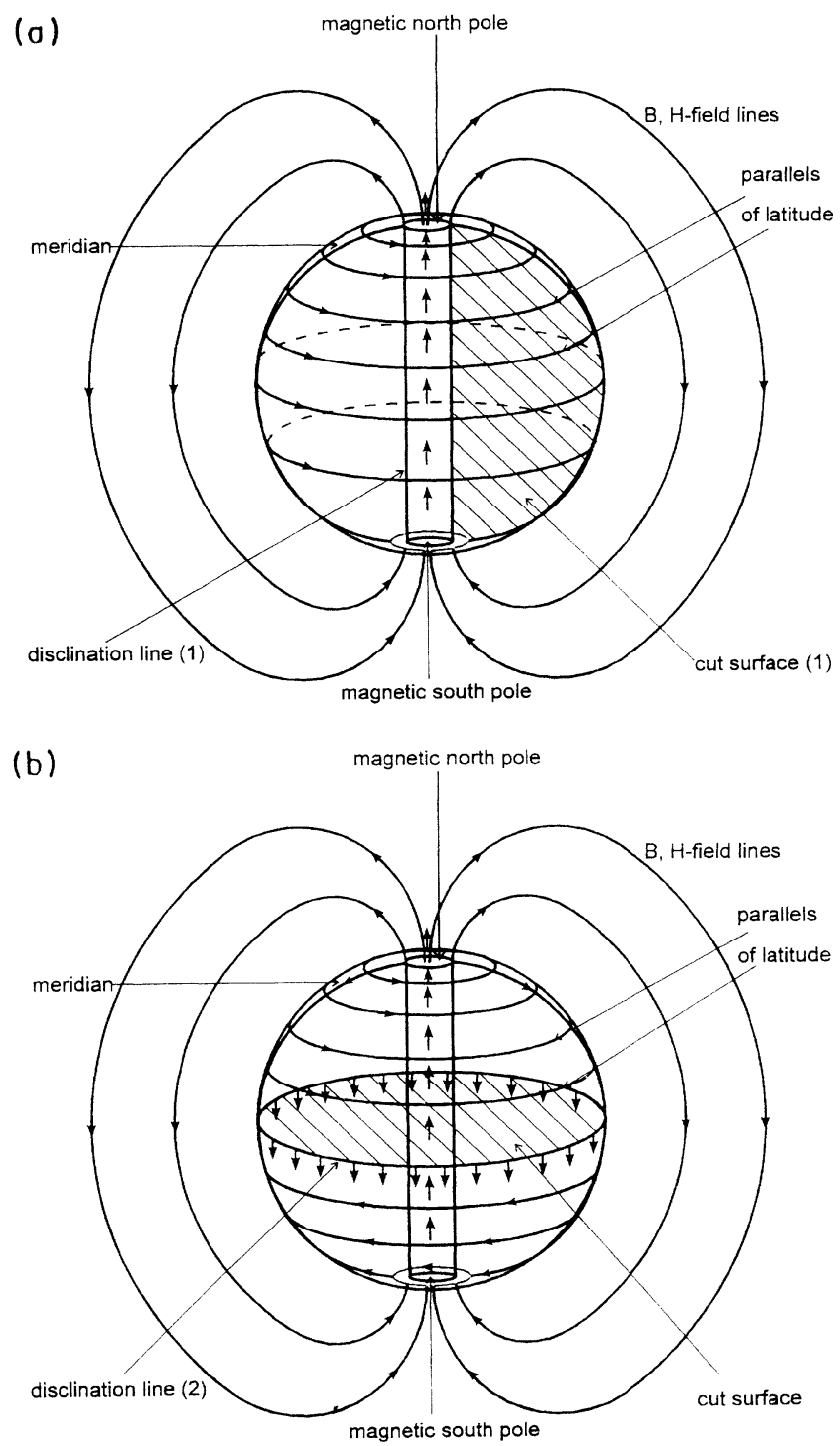

FIG. 2. Schematic representation of order-parameter and magnetic-field line configuration for a solid ball $M=\mathscr{B}$ without applied magnetic field $\mathbf{H}$. The surface $\left(\partial_{e} \mathcal{M}\right)$ of $\mathcal{B}$ is a magnetic surface $[B \cdot n(r)=0]$ with the exception of spherical caps at the north and south poles. (a) Simplest magnetic configuration $\left(Q_{\mathscr{H}}=0\right)$ where arrows indicate the $\mathbf{N}(r)$ field, and disclination (1) connects north and south pole. (b) Nontrivial magnetic configuration $\left(Q_{\mathscr{H}} \neq 0\right)$ obtained from (a) by adding disclination (2). Along parallels of latitude only the azimuthal component and along the meridian and disclination line (2) only the radial component of $\mathbf{N}(\mathbf{r})$ is drawn with respect to north pole. Notation for disclination (1) and cut surface (1) of (a) is suppressed. 
respect to the uniformly magnetized sphere $(i)$ of radius $R$ and $H=0$,

$\mathscr{H}^{(f)} / \mathscr{H}^{(i)} \cong\left[9 /\left(\kappa_{S} R\right)^{2}\right] \ln \left(\frac{2 R}{e r_{c}}\right)+O\left[1 /\left(\kappa_{S} R\right)^{2}\right]$,

where $r_{c} \cong \pi \kappa_{S}^{-1}$ represents the core radius, lne $=1$, and the second term refers to the core energy. For the case illustrated in Fig. 2(b) the right-hand side will be multiplied by about a factor 2. Thus for $\kappa_{S} R \gg 1$, and $\kappa_{S}^{-1} \ll \kappa_{H}^{-1}$, it is energetically favorable up to about an $n$ fold "iteration" of the configuration indicated in Fig. 2(a) [with $n=2$ for Fig. 2(b)], and $n \lesssim \mathscr{H}^{(i)} / \mathscr{H}^{(f)}$ to replace the uniform configuration by a nonuniform one. In that case we have $\mathbf{n}(\mathbf{r}) \cdot \mathbf{M}(\mathbf{r})=0$ almost everywhere on $\partial_{e} \mathcal{M}$, and where $n(r)$ is the normal vector to $\partial_{e} \mathcal{M}$ at $r$. The well-known (no-hair) theorem says, however, that a two sphere to which $\partial_{e} M$ is homeomorphic cannot be combed, ${ }^{11,12}$ i.e., there must exist defects of the vortex type occurring in pairs [see, e.g., Figs. 2(a) and 2(b)].

\section{B. Topology of defects}

A characterization of the defects in the Heisenberg model (6) for $J(r) \equiv J$ can be done via the homotopy groups $^{11,12}$

$$
\begin{aligned}
& \pi_{1}\left(S^{2}\right)=I, \\
& \pi_{2}\left(S^{2}\right)=\mathbb{Z}, \\
& \pi_{3}\left(S^{2}\right)=\mathbb{Z} .
\end{aligned}
$$

Here $S^{2}$ represents the two sphere and corresponds to the order-parameter space, i.e., $\mathrm{N} \in S^{2}$, and $\mathrm{Z}$ represents the infinite cyclic group. From (13) follows that the system may contain defects of the "magnetic" $N$-pole type in the bulk and at the surface [see Fig. 2(a)] as explained further below, and (14) refers to an infinite variety of bulk textures, which may be displayed by the order parameter. Although (12) implies that there exist no stable line defects in such systems, it follows from (14) that entangled "line defects" may be stable and represent the dominant nonlinear structures of the order parameter. ${ }^{13-15}$ For an example, see Fig. 2(b). Line defects in the following will be called disclinations and have, in fact, a tubelike core structure of radius $r_{c} \cong \pi \kappa_{S}^{-1}$. They can be characterized by oriented lines, which play an auxiliary role for the coordination of the defect structure and a disclination strength $S \in \mathbb{Z}$, similarly to a vortex loop in superfluid helium 4, except that they do not have a singular core because the order parameter is $S^{2}$ and not $S^{1}$ (for details see Ref. 15).

The property that point singularities in the bulk arise for a nontrivial realization of (13) [having large energies due to (5a) and (6)] and that the surface texture is also classified by (13) is explained as follows. Surface texture corresponds to a map of the surface $\left(\partial_{e} \mathcal{M}\right.$ ) (being homeomorphic to $S^{2}$ ) into the order-parameter space (i.e., the magnetization), which is also homeomorphic to $S^{2}$. Because of (13), an infinite number of topologically different surface textures exists. Taking account of the boundary conditions for the magnetization at the surface for $\kappa_{S}^{-1} \ll \kappa_{H}^{-1}$ implies a map from $S^{2}$ to $S^{1}$ (almost everywhere), but this does not change the texture and only implies that the surface "singularities," i.e., the magnetic $N$-poles, appear in pairs. Point singularities of the order parameter in the bulk are also classified by (13) because one only needs to put a surface $S^{2}$ around the point defect and study the map $S^{2} \rightarrow S^{2}$ in the same fashion as explained above for $\partial_{e} \mathcal{M}$. In contrast to the latter case, no boundary conditions exist because the area of the two sphere around the defect can be arbitrarily small, and therefore magnetic $N$-poles come into existence.

Let us point out that the magnetic-field lines in Fig. 2(a) have been drawn in such a fashion that they enter and leave the solid ball $\mathcal{B}$ of radius $R$ at the magnetic south and north pole, respectively, through small spherical caps $\left(\delta S_{i}^{2}\right)$. Accordingly, the rest of the surface $S^{2 \prime}=S^{2}-\left\{\delta S_{i}^{2}\right\}$ has the property of a magnetic surface, i.e., $\mathbf{n} \cdot \mathbf{B}=0$, where $\mathbf{n}$ is normal to the surface $S^{2 \prime}$. It is obvious, however, that for some field $\{\mathbf{N}(\mathbf{r})\}$, satisfying $\mathrm{n} \cdot \mathrm{N}=0$ along $S^{21}$, this does not imply that $S^{21}$ is a magnetic surface, or vice versa. On the other hand, it can be assumed that for $\kappa_{S}^{-1} \ll R$ and an equilibrium configuration $\{\mathbf{N}(\mathbf{r})\}_{e}$ the torque of the magnetic field at $S^{21}$ will vanish in order to keep the magnetic surface charge as low as possible. In the interior of $\mathcal{B}$ the surface charge is then diluted and redistributed in such a fashion that within a multipole expansion of the first term of (5b) all multipoles vanish. For the bulk dipole and quadrupole moments this implies, respectively,

$$
\begin{aligned}
& \mathbf{M}=-\int_{\mathcal{M}} d^{3} x \times \nabla \cdot \mathbf{M}(\mathbf{x})=0, \\
& \mathbf{Q}=-\int_{\mathcal{M}} d^{3} x\left(3 \times x-x^{2} \underline{I}\right) \nabla \cdot \mathbf{M}(\mathbf{x})=0,
\end{aligned}
$$

but depending on the magnitude of $R \kappa_{S}<\infty$, it will fail to apply to large-order multipoles. Similar considerations apply to Fig. 2(b) and generalizations, where $n>1$ pairs of magnetic surface poles exist corresponding to the representations of the group $\pi_{2}\left(S^{2}\right)$. Also in that case, $n<\left(R \kappa_{S}\right)^{2} / \ln \left(R \kappa_{S}\right)$, as derived from (11), is necessary, but a precise upper bound of $n$ as a function of $R \kappa_{S}$ has not been derived. Note that the remanent magnetization $M_{R}$ of a sample depends on the "area" of the external surface covered by magnetic surface poles added up according to their strength.

It should be pointed out that the present problem is related to the defect structure in liquid crystals, which has been extensively studied from a topological point of view by Bouligand ${ }^{16}$ and Bouligand et al. ${ }^{17}$ There the order parameter is the projective sphere $P^{2}$, and (12) is replaced by $\pi_{1}\left(P^{2}\right)=\mathbb{Z}_{2}$, whereas (13) and (14) are identical. Due to $\pi_{1}\left(P^{2}\right)=\mathbb{Z}_{2}$ there exist topological stable disclinations of strength $S=\frac{1}{2}$ and $S=-\frac{1}{2}$, which can be transformed into each other. Disclinations of integer strength, however, are unstable as they may dissociate via one of the following two processes: $S \rightarrow \frac{1}{2}+\frac{1}{2}$ or $S \rightarrow \frac{1}{2}-\frac{1}{2} \rightarrow 0$, applying to $S=1$. The first process can always take place, due to energetic reasons, independent of how entangled the disclination is, whereas the second process requires a large configurational change and is forbidden for entan- 
gled configurations characterized by a nontrivial element of $\pi_{3}\left(P^{2}\right)$. The $S^{2}$ order-parameter dissociation into disclinations of strength $S \in \mathbb{Z}+\frac{1}{2}$ is forbidden, and therefore the texture group $\pi_{3}\left(S^{2}\right)$ stabilizes disclinations of integer strength.

It should be emphasized that the classical Heisenberg Hamiltonian and its continuum approximation (6) apply in the limits where the spin $(s)$ and the temperature $(T)$ obey asymptotically $s \rightarrow \infty, T \rightarrow 0$. Although itinerant magnets on a quantum-mechanical level cannot be represented by a Heisenberg model, the expectation value $\langle\mu\rangle$ of magnetic moments is well defined and implies, in $\mathrm{Fe}, \mathrm{Co}$, and $\mathrm{Ni},\langle\mu\rangle \sim \mu_{B} \mathrm{O}(1)$. Accordingly, we are far away from the domain where a semiclassical approximation applies, implying that high-energy (short-range) phenomena, which are dominated by the correlation length $\xi$, need an extension of the micromagnetic formalism, as indicated in Sec. III.

\section{Hopf's invariant and Gauss' linking number}

A quantitative measure of (14) is Hopf's invariant, ${ }^{12}$ also called magnetic helicity by Berger and Field, ${ }^{18}$ which can be represented in the form given by Kundu and Rybakov, ${ }^{14}$

$$
Q_{\mathscr{H}}=-\frac{1}{(8 \pi)^{2}} \int_{\mathbf{R}^{3}} d^{3} r \mathbf{A}(\mathbf{r}) \cdot \mathbf{B}(\mathbf{r}),
$$

where $\mathbf{A}$ and $\mathbf{B}$ are vector potential and magnetic induction field, respectively. Supposing that $\mathbb{R}^{3}-\mathcal{M}$ is a vacuum; it may also be represented in the form ${ }^{15}$

$$
\begin{aligned}
Q_{\mathcal{H}}= & \frac{2}{(8 \pi)^{3}} \int_{\mathcal{M}} d^{3} r \int_{\mathcal{M}^{\prime}} d^{3} r^{\prime} \frac{\mathbf{B}(\mathbf{r}) \cdot\left[\left(\mathbf{r}-\mathbf{r}^{\prime}\right) \times \mathbf{B}\left(\mathbf{r}^{\prime}\right)\right]}{\left|\mathbf{r}-\mathbf{r}^{\prime}\right|^{3}} \\
& -\frac{2}{(8 \pi)^{3}} \int_{\mathscr{M}} d^{3} r \int_{\partial^{\prime} M} d^{2} \mathbf{r}^{\prime} \frac{\mathbf{B}(\mathbf{r}) \times \mathbf{B}\left(\mathbf{r}^{\prime}\right)}{\left|\mathbf{r}-\mathbf{r}^{\prime}\right|} .
\end{aligned}
$$

From this it follows that $Q_{\mathscr{H}}$ is a pseudoscalar. The first term of (16) is proportional to the Gaussian linking number and the second term results from the presence of surfaces. An equivalent expression to (16) can be obtained via the replacement ${ }^{15}$

$$
B(r) \rightarrow 4 \pi M(r) \text {. }
$$

In the case $\partial^{\prime} M=\emptyset,(16)$ can also be expressed in terms of the Gaussian linking number $\Phi\left(\partial C_{a}, \partial C_{b}\right)$,

$$
\Phi\left(\partial C_{a}, \partial C_{b}\right)=\frac{1}{4 \pi} \int_{\partial C_{a}} d x^{i} \int_{\partial C_{b}} \frac{d y^{j} \epsilon_{i j k}(\mathbf{x}-\mathbf{y})^{k}}{|\mathbf{x}-\mathbf{y}|^{3}}
$$

in the form

$$
Q_{\mathcal{H}} \sim \sum_{a<b} S_{a} S_{b} \Phi\left(\partial C_{a}, \partial C_{b}\right)
$$

Here $\partial C_{a}$ represents a closed loop corresponding to the ath disclination of strength $S_{a}$. The loop may be viewed as the oriented boundary of the cut surface $C_{a}$ suspended by the disclination loop $\partial C_{a}{ }^{15}$

Consider next the case that $\mathbf{M}(\mathbf{r})$ for $\mathbf{r} \in \partial_{e} \mathcal{M}$ is tangential to $\partial_{e} \mathcal{M}$, almost everywhere, i.e., $\kappa_{S}^{-1}<<\kappa_{H}^{-1}$. Singularities appear there, where disclination lines pierce through $\partial_{e} \mathcal{M}$. The simplest situation arises if $\mathcal{M}$ is a three-ball $\mathscr{B}$ and there is just one disclination of strength $S$ extending from the south to the north pole of $\mathcal{B}$ [see Fig. 2(a)]. At points where the disclination pierces through the surface, it leaves magnetic poles, as a consequence of (13), of strength $\sim \pm S$ depending on orientation and with a linear size of the order of $S \kappa_{S}^{-1}$. In case that the tubelike structure of the core of a $S=1$ disclination is tentatively considered as a radial Néel-type wall the size of the pole would be $\pi \kappa_{S}^{-1}$. Note that Hubert ${ }^{19}$ has studied such polelike structures in a soft magnetic material, calling them "swirls," having a linear dimension $\sim \pi \kappa_{S}^{-1}$. Different configurations in Fig. 2(a) (without moving the disclination) can be obtained via local $\mathrm{SO}(2)$ transformations of the order parameter on the two sphere, whereas a displacement of the disclination can be obtained via a Möbius transformation of $S^{2}$. The disclination considered can be moved around in $\mathcal{B}$ by an applied magnetic field but cannot be easily driven out of $\mathcal{B}$ as long as $\kappa_{H}^{-1} \gg \kappa_{S}^{-1}$. The situation is different for disclination (2) in Fig. 2(b), which may be annihilated either by contraction through disclination (1) or by sliding it up or down to the poles and expelling it.

Recall that the only one-to-one rational transformations of the Riemann sphere $\left(S^{2}\right)$ into itself are the fractional Möbius transformations

$$
z \rightarrow \Phi(z)=\frac{a z+b}{c z+d}, \quad a d-b c \neq 0,
$$

where $z, a, b, c$, and $d$ are complex numbers and $z$ is obtained from $S^{2}$ by stereographic projection. It can be regarded as a coordinate transformation in the sphere $S^{2}$ and therefore provides for a singularity-free displacement of the surface structure of magnetization (the magnetization is dragged along by the coordinate transformation). The latter may be considered as a boundary condition for the magnetization in the bulk. An even more plastic picture arises if the three-ball $\mathscr{B}$ is supposed to be filled with a ferrofluid subject to the same boundary condition at $S^{2}=\partial \mathcal{B}$. Next one imposes flow structures to the ferrofluid, characterized by velocity fields $\{\mathbf{v}(\mathbf{r})\}$, which are singularity free at the surface $S^{2}$. This implies that the magnetization is dragged along by the flow (corresponding to coordinate transformations). In addition, the helicity $Q_{\mathscr{H}}^{\prime}$ of the flow field $\{\mathbf{v}(\mathbf{r})\}$ can also be computed by (15) using the replacements $\mathbf{A}(\mathbf{r}) \rightarrow \mathbf{v}(\mathbf{r})$ and $\mathbf{B} \rightarrow \boldsymbol{\nabla} \times \mathbf{v}$. Thus any singularity-free transformation of the magnetization structure will leave $Q_{\mathscr{H}}$ and $Q_{\mathscr{H}}^{\prime}$ invariant.

A representation similar to (18) of (16) for $\partial^{\prime} M \neq \emptyset$ is not known to the authors, but the problem is studied in much detail by Berger and Field ${ }^{18}$ to whom we refer for a more sophisticated treatment of this problem. Suppose, for the sake of simplicity, that (16) and (18) also apply to (15) when $\mathbb{R}^{3}$ is replaced by $\mathbb{R}^{3} \cup \infty \cong S^{3}$ (three-sphere) and that $\partial^{\prime} M=\partial_{e} M$ applies. Then (18) consists essentially of three terms,

$$
Q_{\mathscr{H}}=Q_{M_{M}}+Q_{M \bar{M}}+Q_{\bar{M} \bar{M}},
$$

where $\bar{M}$ is the complement of $M$ in $S^{3}$. The terms $Q_{M M}$ 
and $Q_{\bar{M} \bar{M}}$ refer to entanglements of disclinations and magnetic flux tubes being completely located inside $M$ and inside $\bar{M}$, respectively. $Q_{\bar{M} \bar{M}}=0$ is reasonable because even if the system expels helicity through $\partial_{e} M$ it will dissipate to nothing if $\bar{M}$ does not represent an ideal magnetohydrodynamic plasma (vanishing electrical resistance). The term $Q_{M \bar{M}}$ refers to pairs of loops, where at least one partner has support in $M$ and $\bar{M}$ simultaneously. In order to represent $Q_{M \bar{M}}$ in the form (18) we suppose that a disclination piercing through two points of $\partial_{e} \mathcal{M}$ is closed via a fictitious disclination segment, represented, e.g., by one line of the B field in Fig. 2(b). Entanglements between fictitious disclination segments will be fixed once and for all after they come into existence and, therefore, will play no role in the following. This idea is very close to the concept of relative helicity developed by Berger and Field. ${ }^{18}$ These authors also distinguish between external and internal helicity flux tubes. Internal helicity is related to a self-linking of a loop and corresponds to diagonal terms in (20). In the present formalism self-linking can be represented by a number of small ring-shaped disclination loops sliding along the main loop ${ }^{15}$ and is therefore included in (18), where diagonal terms vanish.

For a NS ferromagnet we have $\partial^{\prime} \mathcal{M}=\partial_{e} \mathcal{M}+\partial_{i} \mathcal{} \rho+\partial_{i} \mathcal{L}$ in the simplest case, and, therefore, the second term in (16) can never be ignored and the first term even for $\partial_{e} \mathcal{M}=\emptyset$ cannot be expressed in terms of (20) because $M_{S}(r, T)$ is position dependent. Defining, however, the dimensionless field

$$
m_{S}(\mathbf{r}, T) \equiv M_{S}(\mathbf{r}, T) / M_{S}(T) \geq 0,
$$

where $M_{S}(T)$ is the average saturation magnetization density, (18) may be replaced approximately by

$$
\begin{array}{r}
\Phi^{\prime}\left(\partial C_{a}, \partial C_{b}\right)=\frac{1}{4 \pi} \int_{\partial C_{a}} d x^{i} \int_{\partial C_{b}} \frac{d y^{j} \epsilon_{i j k}(\mathbf{x}-\mathbf{y})^{k}}{|\mathbf{x}-\mathbf{y}|^{3}} \\
\times m_{S}(\mathbf{x}, T) m_{S}(\mathbf{y}, T) .
\end{array}
$$

One may also consider the approximation, where one sets $\partial^{\prime} M=\emptyset$ and uses a smoothed out weight function $m_{S}(\mathbf{r}, T)$. From this follows that $Q_{H_{\mathcal{H}}}$ is not anymore proportional to a link invariant with respect to disclination loops and, therefore, changes continually upon displacement of disclination lines. However, if $\partial C_{a}$ and $\partial C_{b}$ move exclusively in one of the submanifolds of $\mathcal{M}$, i.e., $\mathcal{S}$ or $\mathcal{L}$, then during that time $\left(18^{\prime}\right)$ is a link invariant. In case that $\partial C_{a}$ and $\partial C_{b}$ sweep over $\mathcal{S}$ and $\mathcal{L}$ simultaneously, $\Phi^{\prime}\left(\partial C_{a}, \partial C_{b}\right)$ will oscillate around a time average, which is proportional to $\Phi\left(\partial C_{a}, \partial C_{b}\right)$.

\section{QUASISTATIC CHANGE OF TOPOLOGY}

In the following we will develop a simple method to describe the quasistatic change of topology $\left(\partial Q_{\mathscr{H}} / \partial t=0\right)$ in NS ferromagnets in connection with a phenomenological theory of the coercive force $H_{c}$. The main idea underlying this approach is the hypothesis that $H_{c}$ is a measure of the strength of topological obstruction to which crossing processes of disclinations in a network of disclinations are subject. This requires the introduction of a characteristic time $\eta_{t}$, which has the significance of a nonlocal (in space and time) spin resistivity.

\section{A. Electromagnetics of topological obstruction}

It should be recalled, that the Gaussian linking number is the simplest known knot and link invariant and, besides, that it does not resolve such objects particularly well. A better job in this respect is done by knot polynomials, e.g., the Jones and Kauffman polynomials (see, e.g., Kauffman $\left.{ }^{20}\right)$. The point we want to make is that a given equivalence class of the texture group $\pi_{3}\left(S^{2}\right)$ can be characterized by an entangled structure of oriented disclination lines, which modulo orientation and disclination strength represent a knot or link and, as such, can be characterized by a knot polynomial. The essential point in the following, however, is only to have some qualitative notion of knottedness and linking in analytical form. Even more important is the concept of a crossing process of disclinations, changing their entanglement. For the helicity $Q_{\mathscr{H}}$ and $M \cong S^{3}$ this may be computed over the formula (see, e.g., Ref. 15)

$$
\frac{d}{d t} Q_{\mathscr{H}}=\frac{2 c}{(8 \pi)^{2}} \int_{S^{3}} d^{3} r \mathbf{E} \cdot \mathbf{B}
$$

where $c$ is the speed of the light, and $\mathbf{E}$ is the electric field strength. E and B may now be computed over a retarded solution of Maxwell's equations (10), stated in Sec. II, yielding the nonlocal relation $\mathbf{Y}=\mathbf{Y}\left(\mathbf{H}, \mathbf{M}, \epsilon_{0}, \sigma\right)$ for $\mathbf{Y}=\mathbf{E}$ and $\mathbf{B}$. Here the dielectric and conductive properties of NS material enter in a natural fashion. In a simpler approach one may use a generalization of Ohm's law $^{21}$ (for a quasistatic time change),

$$
\begin{aligned}
\mathbf{E}(\mathbf{x}, t)= & : \eta \mathbf{J}:+: R(\mathbf{B} \times \mathbf{J}): \\
& +: \beta_{1} B^{2} \mathbf{J}:+: \beta_{2}(\mathbf{B} \cdot \mathbf{J}) \mathbf{B}:+\cdots,
\end{aligned}
$$

where $\eta=1 / \sigma$ is the inverse conductivity, $R$ the Hall coefficient, $\beta_{1}$ and $\beta_{2}$ are coefficients of nonlinear terms, and $\mathbf{J}$ is Ohm's current. Furthermore, we have introduced the notation

$$
: \eta \mathbf{J}:=\int_{S^{3}} d^{3} x^{\prime} \int_{-\infty}^{t} d t^{\prime} \underset{\sim}{\eta}\left(\mathbf{x}, t ; \mathbf{x}^{\prime}, t^{\prime}\right) \mathbf{J}\left(\mathbf{x}^{\prime}, t^{\prime}\right)
$$

generalizing the well-known laws to nonlocality in space and time and taking account of their tensor character. Inserting $\mathbf{E}$ into (21) yields

$$
\frac{d}{d t} Q_{\mathscr{H}}=\frac{2 c}{(8 \pi)^{2}} \int_{S^{3}} d^{3} r:\left[\eta+\left(\beta_{1}+\beta_{2}\right) B^{2}\right] \mathrm{J}: \cdot \mathbf{B}
$$

and in linear response theory

$$
\frac{d}{d t} Q_{\mathcal{H}} \cong \frac{2 c}{(8 \pi)^{2}} \int_{S^{3}} d^{3} r: \eta \mathrm{J}: \cdot \mathbf{B} .
$$

From this follows that creation or annihilation of linking of disclinations is only possible if $\eta>0$, i.e., for finite resistance, applies at some finite space or time scales. Observe that the dissipated Joule's energy $\left(W_{J}\right)$ is governed 
by a similar law

$$
\frac{d}{d t} W_{J}=\int_{S^{3}} d^{3} r \mathbf{J} \cdot \mathbf{E}=\int_{S^{3}} d^{3} r: \eta \mathbf{J}: \mathbf{J}
$$

where in the quasistatic approximation $\left(\partial W_{J} / \partial t=0\right)$ the dot operator can be ignored and $\eta=\eta(0,0)$ corresponds to the dc resistivity.

Consider now $d Q_{\mathscr{H}} / d t$ for the space $M$ with $\partial_{e} M \neq \emptyset$ defined in such a fashion that

$$
\frac{d}{d t} Q_{\mathscr{H}}=\frac{d}{d t} Q_{M M}+\frac{d}{d t} Q_{M \bar{M}}
$$

Here each term on the right-hand side (rhs) is computed via crossing processes and a sweeping motion in NS material (see the end of Sec. II) of disclinations in $M$, which yield, after completion,

$$
\Delta Q_{\mathscr{H}}=\Delta Q_{n n}+\Delta Q_{M \bar{M}} .
$$

For the sake of simplicity, the oscillatory contribution to (26) resulting from the sweeping motion of disclinations in NS ferromagnets will be ignored in the following.

Note that not every change of $\mathbf{M}(\mathbf{r}, t)$ will lead to $\Delta Q_{\mathscr{H}} \neq 0$. Because different equivalence classes of $\pi_{3}\left(S^{2}\right)$ can only be reached over singularities of the $\mathbf{N}(\mathbf{r}, t)$ field, a significant change of $Q_{\mathscr{H}}$ is only possible if two disclinations cross each other. In that case no local inertial frame can be found where the $\mathbf{E}$ field vanishes, and therefore $d Q_{\mathscr{H}} / d t \neq 0$ results. However, that does not imply $\Delta Q_{\mathcal{H}} \neq 0$ after completion of the process. Consider for instance the formation of a trefoil knot, for which $Q_{\mathscr{H}}=0$ results because its (external) Gaussian linking number vanishes. Its formation may proceed, e.g., from an unlinked loop $\left(Q_{\mathscr{H}}=0\right)$ via any odd number of selfintersections or from a Hopf link $\left(Q_{\mathscr{H}} \neq 0\right)$ via suitable reconnection intermediately involving two pairs of magnetic $N$ poles, ${ }^{15}$ belonging to representations of the group $\pi_{2}\left(S^{2}\right)$. Accordingly, crossing processes of disclinations are fundamental for any change of structure and are associated with singularities of the $N$ field in a semiclassical continuum approach.

Before we proceed to the derivation of a more practical formula from $\left(21^{\prime}\right)$ some qualitative remarks with respect to the significance of $(21)$ and $\left(21^{\prime}\right)$ will be made. Despite the fact that we here study an essentially magnetic problem of topological defects, any significant change of topology measured by $d Q_{\mathscr{H}} / d t$ involves the electric-field strength $\mathbf{E}$ as a simple consequence of Faraday's law. Accordingly, this law acts as a constraint for the topological degrees of freedom of the system once some initial condition has been fixed. Note that for $\sigma \rightarrow \infty$, in the case of a superconductor, we have $B \equiv 0$ due to the Meissner effect, and, therefore, $\mathbf{E} \cdot \mathbf{B} \equiv \mathbf{0}$. However, even in the case of an ideal conductor (noninteracting electrons) we have $\sigma(0)=\infty$, but $\sigma(\omega) \neq 0$ due to causality (KramersKronig) and therefore $d Q_{g_{\mathcal{H}}} / d t \neq 0$ because $\eta=1 / \sigma$ enters $(21)$ in a nonlocal fashion. Suppose, however, that we invoke the fictitious conductor, characterized by $\sigma(\omega) \equiv \infty$; then all currents are perfectly screened and no electric field develops, i.e., $D=4 \pi \mathcal{P}$. Furthermore, Faraday's law implies $\partial_{t} \mathbf{B}=0$, and therefore any change $(\delta \mathbf{M})$ of $\mathbf{M}$ is transferred into a change $\left(\delta \mathbf{H}^{\prime}\right)$ of $\mathbf{H}+\mathbf{H}_{S}$, i.e., $\delta \mathbf{H}^{\prime}=-4 \pi \delta \mathbf{M}$, implying a winding and stretching of magnetic field lines, with the field energy $\delta \mathscr{H}$ steadily increasing. This implies that with each successive hysteresis cycle more and more energy is stored in the system, and, therefore, no stationary (periodic) state can be reached.

In the opposite case of the fictitious insulator $\sigma(\omega) \equiv 0$, (21') implies $d Q_{\mathscr{H}} / d t \sim \infty \times 0$ but still vanishes because there is no support for $J \equiv 0$. However, for a physical insulator we have $\sigma(0)=0$, but $\sigma(\omega) \neq 0$ due to causality, and, therefore, $d Q_{H_{t}} / d t \neq 0$. Because coercive fields $\left(H_{c}\right)$ of NS insulators and metals may be of the same order of magnitude, it is suggestive that $\left(21^{\prime}\right)$ depends essentially on nonlocal properties of $\eta$.

\section{B. Estimate of $\boldsymbol{\eta}_{\boldsymbol{t}}$ for crossing processes}

Because the crossing process of two disclinations is a local phenomena involving essentially the cores of disclinations of size $r_{c} \sim \pi \kappa_{S}^{-1}$ and a transit time $\tau_{t}$, we may set in $\left(21^{\prime}\right)$

$$
\eta_{t} \equiv \int_{\left(r_{c}\right)^{3}} d^{3} r \int_{0}^{\tau_{t}} d t \eta(\mathbf{r}, t)
$$

and delete the dot operator, i.e., we will set

$$
\frac{d Q_{\mathscr{H}}}{d t}=\frac{2 c}{(8 \pi)^{2}} \eta_{t} \int_{S^{3}} d^{3} r \mathbf{J} \cdot \mathbf{B},
$$

where $\partial Q_{\mathscr{H}} / \partial t=0$ and $\eta_{t}$ represents the spin resistivity. Here we have set $\eta(\mathrm{r}, t)=1 / \sigma(\mathrm{r}, t)$ and assumed spacetime translation symmetry, i.e., $\sigma\left(\mathbf{r}, t ; \mathbf{r}^{\prime}, t^{\prime}\right)$ $=\sigma\left(\mathbf{r}-\mathbf{r}^{\prime}, t-t^{\prime}\right)$. In a more physical approach, we may use instead of (27)

$$
\eta_{t} \equiv \int_{S^{3}} d^{3} r \int_{0}^{\infty} d t \eta(\mathbf{r}, t) K(\mathbf{r}, t)
$$

where $K(\mathbf{r}, t)$ is a normalized transition amplitude, which may be computed at low temperatures via the Pauli-Van Vleck-Morrette formula ${ }^{22}$

$$
\begin{aligned}
K\left(\xi, t ; \xi^{\prime}, t^{\prime}\right)= & \operatorname{det}\left[\frac{1}{2 \pi i} \frac{\partial^{2} S\left(\xi, t ; \xi^{\prime}, t^{\prime}\right)}{\partial \xi \partial \xi^{\prime}}\right]^{1 / 2} \\
& \times \exp \left[(i / \hbar) S\left(\xi, t ; \xi^{\prime}, t^{\prime}\right)\right]+O(\hbar) .
\end{aligned}
$$

Here $S$ satisfies the usual Hamilton-Jacobi equation,

$$
\partial S / \partial t+H^{\prime}(\xi, \partial S / \partial \xi, t)=0 \text {. }
$$

In a model where the topological transition takes place over the intermediate formation of a pair of monopoles (see, e.g., Ref. 15), we have $\xi \equiv\left(\mathbf{r}^{+}, \mathbf{r}^{-}\right)$and can compute $\mathscr{H}^{\prime}$ from $\mathscr{H}+\mathscr{H}_{\dot{S}}$ introduced in Sec. II. Using the boundary conditions $\mathbf{r}^{+}=\mathbf{r}^{-}=\mathbf{r}$ and $\mathbf{r}^{+}=\mathbf{r}^{\prime}=\mathbf{r}^{\prime}$ and the constraint that the topological transition starts at $t$ and is completed at $t^{\prime}$, we can compute

$$
K\left(\mathbf{r}-\mathbf{r}^{\prime}, t-t^{\prime}\right) \equiv K\left(\mathbf{r}, t ; \mathbf{r}^{\prime}, t^{\prime}\right)
$$

using instanton techniques. ${ }^{22}$ Equation $\left(21^{\prime \prime}\right)$ will only be valid if the crossing processes are sufficiently local in 
space and time, i.e., spatial and temporal overlap of crossing processes cannot be described by (21").

From this discussion follows that even for a quasistatic change of topology the nonlocal resistivity $\eta(r, t)$ enters the problem, weighted by a probability functional $K(r, t)$, which depends directly on the magnetic properties encoded in $\mathscr{H}_{\text {and }} \mathscr{H}_{\dot{s}}$ introduced in Sec. II. At higher temperatures, where a combined process of tunneling and thermally activated hopping takes place, similar arguments can be used and the theoretical concepts developed by $\mathrm{Affleck}^{23}$ may be applied. Also related to the present problem is the issue of the sphaleron-induced baryon decay, which is extensively studied in the literature. ${ }^{24}$

In order to derive a simple estimate of the order of magnitude of the activation barrier $\left(\Delta E_{a}\right)$ for a crossing process of two disclinations the following model will be adapted. Consider first a disclination of the type illustrated in Fig. 2(a) with an azimuthal magnetization configuration, which may be represented as $\mathbf{N}=(\cos \varphi, \sin \varphi)$ with $\varphi=+\arctan y / x+\pi / 2$. Similar considerations apply to $\varphi=-\arctan x / y$, which, however, on a sphere produces a quadrupolar surface magnetization. The energy of the azimuthal disclination will be purely of exchange type and of the order of $\mathcal{H}_{J}^{(\mathrm{az})} \cong 2 \pi J l \ln (R / a)$, where $l, R$, and $a$ are axial extension, radial extension of the field $\{\mathbf{N}(\mathbf{r})\}$, and lattice constant, respectively. Obviously the "core" energy of that disclination can be reduced by replacing $a$ by $r_{c} \gg a$ and letting $\{\mathbf{N}(\mathbf{r})\}$ for $r<r_{c}$ turn smoothly into the axial direction. This yields the change of energy $\Delta \mathscr{H}^{(a)}$ of $\mathscr{H}_{J}^{(\mathrm{az})}$

$\Delta \mathscr{H}^{(a)}=\Delta \mathscr{H}_{S}+\Delta \mathscr{H}_{J} \cong \alpha 2 \pi M_{S}^{2}\left(\pi r_{c}^{2}\right) l+\beta 2 \pi J l \ln \left(a / r_{c}\right)$,

where $\alpha$ and $\beta$ depend on $\{\mathbf{N}(\mathbf{r})\}$, and $r_{c}$ is determined from $\partial \Delta \mathscr{H}^{(a)} / \partial r_{c}=0$, as $r_{c} \cong(\beta / \alpha)^{1 / 2} \kappa_{S}^{-1}$. Here we have $\alpha<1, \beta<1$, and $\Delta \mathscr{H}^{(a)}<0$, but need in addition a bootstrap condition between $\alpha$ and $\beta$. The core's energy $\left(\Delta \mathscr{H}_{c}^{(a)}\right)$ can be obtained from (29) by the replacement $\beta \rightarrow \beta-1$. It is, therefore, reasonable to set $\alpha+\beta=1$, and use the boundary conditions to require $(\beta / \alpha)^{1 / 2}=\pi$. This implies $\alpha=1 / \pi^{2}$.

Next we consider the crossing process of two disclinations requiring a modification of $\{\mathbf{N}(\mathbf{r})\}$ over a linear extension $d$ of their respective core tubes. Incompatibility of the two core structures will require an additional energy of the order of

$$
\Delta E_{a} \cong \alpha\left[2 \pi M_{S}^{2}\left(\pi r_{c}^{2}\right) d_{S}+2 \pi J\left(\pi r_{c}^{2} / d_{S}\right)\right] .
$$

Here it has been assumed that $r_{c}$ and $d_{S}$ are the characteristic linear dimensions of the saddle-point configuration, associated with the characteristic magnetostatic and exchange energies, with the weighting factors $\alpha$ and $(1-\beta)$, respectively. From $\partial \Delta E_{a} / \partial d_{S}=0$ follows $d_{S} \cong(2 / \pi)^{1 / 2} r_{c}$ and

$$
\Delta E_{a} \cong \sqrt{2 \pi}\left[1+(\pi / 2)^{1 / 2}\right] J r_{c} .
$$

In a similar fashion one may derive from (29) an estimate of the pinning energy $\Delta E_{p}$. Supposing that the effective linear dimension $\left(\tilde{d}_{\mathrm{NC}}\right)$ over which the core of a disclination is pinned obeys $\bar{d}_{\mathrm{NC}}<\bar{d}_{\mathrm{NC}} \ll 2 r_{c}$, one obtains $\Delta E_{p} \cong(2 / \pi) J \widetilde{d}_{\mathrm{NC}} \ln \left(\widetilde{d}_{\mathrm{NC}} / 2 a\right)$, and $\Delta E_{p}<\Delta E_{a}$. This implies, that at temperatures where $r_{c} \gtrsim d_{\mathrm{NC}}$ applies, pinning will play no more role.

Due to $r_{c} \gg a, \Delta E_{a}$ is large compared to thermal energies at $T<T_{C}$ and therefore the energy barrier cannot be easily overcome by a thermal process. It is obvious, however, that $\Delta E_{a}$ has to be computed over more sophisticated methods and presumably obeys $\Delta E_{a} \approx k_{B} T_{C}$. The point here is that in NS material crossing processes of disclinations can take place at favorable locations of the labyrinthine domain, where $J$ is reduced with respect to the bulk, and in addition has to be averaged over the specimen, relating it to $T_{C}$.

As a simple scaling relation of the transit time $\tau_{t}$ introduced earlier we may set

$$
1 / \tau_{t} \sim\left(1 / \tau_{0}\right)\left(1-T / T_{C}\right)^{x_{t}},
$$

where $x_{t} \cong 1$, and $h / \tau_{0} \gtrsim k_{B} T_{C}$. Here we have used $x_{t}=z v$, where $z$ and $v$ are the dynamic and correlation length $(\xi)$ critical exponents with $z=2, v=\frac{1}{2}$ in meanfield theory. ${ }^{25}$ Equation (32) implies via (27') $\eta_{t} \sim \eta\left(q_{c}, \omega_{c}\right)$, where the right-hand side indicates the Fourier transform with $q_{c} \cong 2 \pi / r_{c} \sim 2 \kappa_{S}$ and $\omega_{c} \cong 2 \pi \tau_{t}^{-1}$, and shows that $\eta_{t}$ differs considerably from its dc value $\eta=\eta(0,0)$. The latter enters essentially in the rate of dissipation $d W_{J} / d t$ (in the quasistatic limit $\partial W_{J} / \partial t=0$ ) caused by a sweeping motion of disclinations.

\section{Phenomenological laws for $H_{c}(T)$}

Suppose now that $Q_{\mathscr{H}}$ holds the status of a thermodynamic (pseudo)potential, i.e.,

$$
Q_{\mathscr{H}}=Q_{\mathscr{H}}(H, M, T)
$$

and that the time change in (21) corresponds to a quasistatic motion along the major $(M, H)$-hysteresis loop. Then one may assume that $d Q_{\mathcal{H}} / d H$ is an odd function of $H, Q_{\mathscr{H}}[ \pm \infty, M( \pm \infty), T]=0$, implying for example

$$
\begin{aligned}
\left.H \frac{d Q_{H_{H}}}{d H}\right|_{ \pm H_{c}} \sim & \left\{\begin{array}{l}
\left(H_{c}^{(1)} / H_{c}\right)^{\alpha_{1}}, \\
{\left[\ln \left(H_{c}^{(2)} / H_{c}\right)\right]^{\alpha_{2}},}
\end{array}\right. \\
& \text { for } M\left( \pm H_{c}\right), \alpha_{1}, \alpha_{2}>0
\end{aligned}
$$

where $H_{c} \geq H_{c}^{(i)}$ applies. The Ansätze above have been constructed under the assumption that the degree of complexity of the network of disclinations is reflected in the ratio of change $d Q_{\mathscr{H}} / d H_{c}$, and that the latter increases with $H_{c}$ diminishing. Within a phenomenological approach it is assumed that the examples (34a) and (34b) apply to different types of disclination entanglements and (or) different temperature regimes of magnetic polarizability properties of the NS material. Within the quasistatic approximation we can set $\partial Q_{\mathcal{H}} / \partial t=0$, and use $d Q_{\mathscr{H}_{t}} / d t=(d H / d t)\left(d Q_{\mathscr{H}_{\mathcal{H}}} / d H\right)$ and $\left(21^{\prime}\right)$ [with $\eta_{t}$ defined by $\left.\left(27^{\prime}\right)\right]$ in the form 


$$
\frac{d Q_{\mathcal{H}}}{d t} \sim\left(\frac{\eta}{\eta^{(i)}}\right) \frac{d \ln H}{d t}\left[a(H) \operatorname{sgn}\left(\frac{d H}{d t}\right)-b(H)\right] .
$$

For $a(H)$ and $b(H)$ are odd and even functions of $H$, respectively, and $\int_{-\infty}^{\infty} d H b(H)=0$ [as follows from $Q_{\mathscr{H}_{\mathcal{H}}}( \pm \infty, M( \pm \infty), T)=0$ ], one obtains for the examples (34)

$$
\frac{H_{c}}{H_{c}^{(i)}} \sim\left\{\begin{array}{l}
\left(\eta_{t}^{(1)} / \eta_{t}\right)^{1 / \alpha_{1}} \\
\exp \left[-\left(\eta_{t} / \eta_{t}^{(2)}\right)^{1 / \alpha_{2}}\right]
\end{array}\right.
$$

Here $H_{c}^{(i)}, \eta^{(i)}$ with $\eta_{t}^{(i)} \leq \eta_{t}$ and $\alpha_{i}$ are constants for $T$ far away from the Curie temperature $T_{C}$. The signum function in (35) provides for a discontinuity of $d Q_{\mathcal{H}} / d t$ at return points $(d H / d t=0)$ of the magnetic field, which is typical for hysteresis phenomena.

Note that under the plausible assumption that $d \eta_{t} / d T>0$ applies, it follows from (36) that $H_{c}$ diminishes with increasing temperature. A change over from the law (34a) to (34b), or vice versa, is considered to be a consequence of a structural change of the network of disclinations as indicated below (34). It is also obvious that such a changeover cannot only be reflected in the $T$ dependence of $\eta_{t}$, because by construction this quantity depends essentially on local (crossing) processes, whereas (21) is a global quantity.

Due to the chiral character of $Q_{\mathscr{H}}$ one may suspect that in the thermodynamic limit for the ensemble average ( $N \rightarrow \infty$ copies of the system considered), $\left\langle Q_{\mathscr{H}_{H}}\right\rangle \equiv 0$ is natural and only for the variance $\left\langle Q_{\mathscr{H}}^{2}\right\rangle \neq 0$ applies, and therefore (36) does not hold, whereas $\left\langle Q_{\mathscr{H}}\right\rangle \neq 0$ would be a consequence of symmetry breaking, taking place upon relieving the constraints $H= \pm \infty$. Because usually $|H|<\infty$, symmetry breaking may be fixed once for all as long as $T<T_{c}$, and it is more appropriate to consider instead of the ensemble individual samples.

Consider now some more general properties of hysteresis loops. In Fig. 3 a few characteristic minor hysteresis loops are schematically drawn, which show a return point memory at the points $A, B$, and $C$, a property that can be explained by means of Preisach models of hysteresis. ${ }^{26}$ Systems that do not show this property have fading memory, i.e., cycling the magnetic field $H$ through a given interval $\left[H_{\min }, H_{\max }\right]$ does not produce closed curves in the $H$-M plane. Preisach models are usually based on a distribution of elementary hysteresis loops with upper and lower switching fields. Presumably a similar model can be constructed from a hierarchical model of knots and links, based on a mapping of such objects onto elementary hysteresis loops. Upper and lower switching fields have then to be brought in connection with the properties of knots and links parametrized, e.g., by polynomial invariants. From Fig. 3 also follows that the properties of a state at a certain point in the $H-M$ plane, e.g., point $B$, do not depend only on its coordinates but on a whole hierarchy of parent states. Within our model we can assume that the latter states have as a

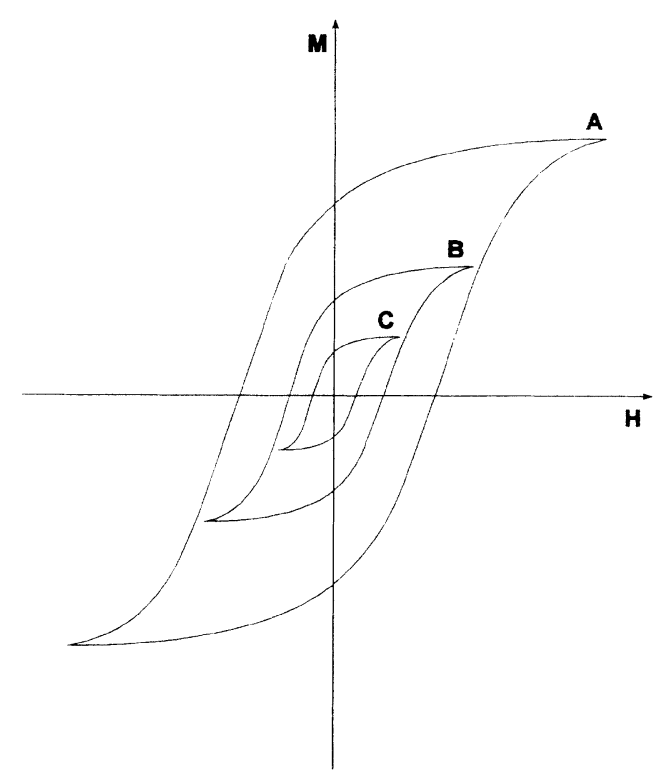

FIG. 3. Schematic representation of minor hysteresis loops showing the property of return point memory indicated by $A$, $B$, and $C$.

representative a precise knot or link invariant. The set of possible states $\mathcal{P}$, e.g., at a point $B=(H, M)$ according to the hypothesis (34) may then be characterized in a very restrictive model by the invariant $Q_{\mathcal{H}}(B, T)$, i.e., all members of the set $\mathcal{P}$ have to have the same Gaussian linking number. On the other hand one may also define $\left\langle Q_{\mathcal{H}}(B, T)\right\rangle_{\mathcal{P}}$ as an ensemble average over the set of states $\mathcal{P}$ at point $B$, in case our hypothesis does not apply. In that case $\left\langle Q_{\mathscr{H}}(B, T)\right\rangle_{\mathcal{P}}=Q_{\mathscr{H}}(H, M, T)$ is a thermodynamic potential but does not satisfy (21) anymore, whereas

$$
\begin{aligned}
d / d t\left\langle Q_{\mathscr{H}}(B, T)\right\rangle_{P} \sim & \left(\eta_{t} / \eta_{t}^{(i)}\right) d \ln H / d t \\
& \times[a(H) \operatorname{sgn}(d H / d t)-b(H)]
\end{aligned}
$$

still applies, and consequently also the arguments leading to (36).

Similar concepts may also be applied to a computation of the remanent magnetization $M_{R}$, simply by the replacements of $H_{c}, H_{c}^{(i)}, M$, and $\alpha_{i}$ in (34) and (36) by $M_{R}$, $M_{R}^{(i)}, H$, and $\beta_{i}$, respectively. There is no reason to replace $\eta_{t}$ and $\eta_{t}^{(i)}$ because by construction these are local quantities, whereas the global laws (34) may depend on the applied field $H$ implying the replacement $\alpha_{i} \rightarrow \beta_{i}$. A simple extension of the theory to minor hysteresis loops can be done formally by the replacement $\left(H_{c}, M_{R}\right)$ $\rightarrow\left(H_{c}^{\prime}, M_{R}^{\prime}\right)$ in our formulas, where the primed coordinates are defined with respect to the barycenter of the minor loop. Such a model has the property of return point memory, and shows discontinuities of $d Q_{H_{t}} / d t$ at return points of the magnetic field. We like to point out that the assumptions underlying the constructions of $d Q_{\mathcal{H}} / d H$ implied by (21) are also satisfied for their application to the computation of properties indicated above. For instance for the minor symmetric hysteresis loops 
$\left(H_{c}^{\prime}, M_{R}^{\prime}\right)$ the degree of complexity of the magnetization configuration must increase with $H_{c}^{\prime}, M_{R}^{\prime} \rightarrow 0$. Using the area of the hysteresis loops as a measure of the energy $W_{J}^{(i)}$ dissipated per cycle, in the quasistatic approximation we obtain with (36)

$$
W_{J}^{(i)}=\oint^{\prime} \mathbf{H} \cdot d \mathbf{M} \sim H_{c}^{\prime} M_{R}^{\prime} \sim H_{c}^{\left(1+\alpha_{i} / \beta_{i}\right)} .
$$

Because in conventional ferromagnets $W_{J}^{(i)}$ obeys power laws with exponents depending on $H^{\prime} \equiv\left|H_{\max }-H_{\min }\right|$, e.g., $\alpha_{i} / \beta_{i}=2$ and 0.6 for small (Rayleigh's law) and large (Steinmetz' law) amplitudes (see, e.g., Bozorth ${ }^{27}$ ), respectively, it can be expected that also in NS ferromagnets $\alpha$ and $\beta$ will depend on $H$.

\section{LOW-ENERGY EXCITATIONAL MODES}

In the following we will outline a qualitative theory of the structure of collective excitations in NS ferromagnets based on the exchange and magnetostatic (stray field) energy. In an ideal Heisenberg magnet without topological defects and vanishing internal fields the corresponding modes have the dispersion $\omega \sim k^{2}$ for $k \rightarrow 0$, have transverse polarization, an infinite transverse correlation length $\xi_{t}$, and are called Goldstone modes. ${ }^{25}$ Longitudinal excitations of the order parameter have a finite correlation length $0<\xi<\kappa_{S}^{-1}$ for $T<T_{C}$ and become of only low energy for $T \rightarrow T_{C}$. They will not be studied in detail here because they are a consequence of thermal production and excitation of topological defects, which is beyond the scope of the present paper. Furthermore, no explicitly time-dependent effects as, for instance, the relaxation of modes will be studied although this is an important subject, since it affects the quasiparticle nature of the excitations (see Sec. VII).

Suppose for the sake of simplicity that the internal field $\mathbf{H}_{i}=\mathbf{H}+\mathbf{H}_{s}$ vanishes. Recall that a uniform magnetization in an ellipsoidally shaped specimen in the absence of crystal anisotropy is unstable against long-wavelength (dipolar) excitations as long as the demagnetization field $\left(H_{s}\right)$ supercedes the applied field $(H)$. This is the reason for the nonuniform magnetization in a NS ferromagnet for $H=0$ in macroscopic samples of any shape with the exception of thin whiskers. The stray field energy $\mathscr{H}_{s}$ can then be computed over the interaction energy and selfenergy, respectively, of a set of dipoles $\left\{\mathbf{p}_{i}\right\}$

$$
\mathscr{H}_{s}=\frac{1}{2} \sum_{i \neq j} W_{i j}+\frac{2 \pi}{3} \sum_{i} \mathbf{p}_{i}^{2} / \Omega_{i}
$$

Here $\Omega_{i}$ is the volume attributed to a dipole, and

$$
W_{12}=\left[\mathbf{p}_{1} \cdot \mathbf{p}_{2}-3\left(\mathbf{p}_{1} \cdot \hat{\mathbf{r}}_{12}\right)\left(\mathbf{p}_{2} \cdot \hat{\mathbf{r}}_{12}\right)\right] / \mathbf{r}_{12}^{3}
$$

is the interaction energy of dipoles at the points $\mathbf{r}_{1}$ and $\mathbf{r}_{2}$. Furthermore, $r_{12}=\left|\mathbf{r}_{1}-\mathbf{r}_{2}\right|$ and $\hat{\mathbf{r}}_{12}=\mathbf{r}_{12} / r_{12}$.

In order to compute the sum in $\mathscr{H}_{s}$ we assume a regular densely packed arrangement of dipoles in a volume of linear dimension $\xi_{n d}$, each dipole being confined to a volume unit $\Omega$ and $\xi_{n d} / \Omega^{1 / 3} \gg 1 . \xi_{n d}$ is a characteristic length for the macroscopic order parameter $N(r)$, which is supposed to be aligned over a distance $\xi_{n d}$ defined via $\left[\left\langle\mathbf{N}(\mathbf{r}) \cdot \mathbf{N}\left(\mathbf{r}+\xi_{n d}\right)\right\rangle \sim N(r)^{2}\right]$, so that the modes obeying $k \xi_{n d}>1$ represent simple magnon excitations. $\xi_{n d}$ can be identified as one of a set $\left\{\xi_{n d}^{i}\right\}$ of characteristic length units of a network of disclinations. Setting $A_{0} \equiv \sum_{j \neq 0} W_{0 j}$ we obtain after Fourier transformation $\left\{\mathbf{p}_{i}\right\} \rightarrow\{\mathbf{p}(\mathbf{k})\}$,

$$
\begin{aligned}
A_{0}(\mathbf{k})= & \frac{4 \pi}{\Omega}\left[\frac{\sin \left(k \xi_{n d}\right)-\left(k \xi_{n d}\right) \cos \left(k \xi_{n d}\right)}{\left(k \xi_{n d}\right)^{3}}-1 / 3\right] \\
& \times\{\mathbf{p}(\mathbf{k}) \cdot \mathbf{p}(-\mathbf{k})-3[\mathbf{p}(\mathbf{k}) \cdot \hat{\mathbf{k}}][\mathbf{p}(-\mathbf{k}) \cdot \hat{\mathbf{k}}]\} .
\end{aligned}
$$

The "excitation" energy $\delta \mathscr{H}^{\prime}(k)$ (in the thermodynamic sense) attributed to the volume unit $\Omega$ and a mode of wave vector $k$, may be represented in the form

$$
\begin{aligned}
\delta \mathscr{H}^{\prime}(\mathbf{k})= & J \Omega k^{2} \mathbf{n}(\mathbf{k}) \cdot \mathbf{n}(-\mathbf{k})+\frac{2 \pi}{\Omega}[\mathbf{p}(\mathbf{k}) \cdot \hat{\mathbf{k}}][\mathbf{p}(-\mathbf{k}) \cdot \hat{\mathbf{k}}] \\
+ & \frac{2 \pi}{\Omega}\left[\frac{\sin \left(k \xi_{n d}\right)-k \xi_{n d} \cos \left(k \xi_{n d}\right)}{\left(k \xi_{n d}\right)^{3}}\right] \\
& \times(\mathbf{p}(\mathbf{k}) \cdot \mathbf{p}(-\mathbf{k})-3[\mathbf{p}(\mathbf{k}) \cdot \hat{\mathbf{k}}][\mathbf{p}(-\mathbf{k}) \cdot \hat{\mathbf{k}}]) .
\end{aligned}
$$

Here the first term refers to the exchange energy, where $\mathbf{n}(\mathbf{k})$ is the Fourier transformation of the field $\mathbf{N}(\mathbf{r})$. In the regime $k \xi_{n d} \gg 1$, the third term above vanishes and the dipolar excitations are described by the second term, which is dispersionless but anisotropic. In the regime $k \xi_{n d} \lesssim 1$, which has no physical significance within the present model (because it implies $\lambda>\xi_{n d}$ ) we have

$\delta \mathscr{H}^{\prime}(k) \cong J \Omega k^{2} \mathbf{n}(\mathbf{k}) \cdot \mathbf{n}(-\mathbf{k})+\frac{2 \pi}{3 \Omega}[\mathbf{p}(\mathbf{k}) \cdot \mathbf{p}(-\mathbf{k})]$.

The modes obeying $\lambda>\xi_{n d}$ represent excitations of the network of disclinations and will depend on the whole set $\left\{\xi_{n d}^{i}\right\}$ of characteristic lengths. In contrast to that, for the modes obeying $\xi_{n d} k \gg 1$, and propagating undamped, $\xi_{n d}$ represents a phase coherence length. Observing

$$
(\sin x-x \cos x) / x^{3} \leq \frac{1}{3}
$$

and the reality properties of $\{\mathbf{N}(\mathbf{r})\}$ and $\left\{\mathbf{p}_{i}\right\}$, $\mathbf{n}(-\mathbf{k})=\mathbf{n}^{*}(\mathbf{k}), \mathbf{p}(-\mathbf{k})=\mathbf{p}^{*}(\mathbf{k})$ (complex conjugate), it follows from (42) and the form of (40) that the dipolar modes within the present model are stable excitations, but that for small wave vectors their magnetostatic energy may become of the same order of magnitude as their exchange energy.

Let us recall that Luttinger and Tisza ${ }^{28}$ have shown that for point dipoles on bcc and fcc lattices the ferromagnetic state has lowest energy, whereas on a sc lattice it is the antiferromagnetic state. For the ferromagnetic state they obtain from the first term on the rhs of (38) the ground-state energy $E_{S}^{0}=-(2 \pi / 3) \sum_{i} p_{i}^{2} / \Omega_{i}$. From this one may expect that for a stochastically and densely packed array of dipoles the dipolar ground state should still be ferromagnetically aligned. From (41) follows, however, that our method yields a vanishing (dipolar) ground-state energy, because the second term on the rhs of (41) corresponds to the self-energy added to (38). If 
that is only an artifact of the Fourier transformation, applied for the sake of simplicity to a uniform distribution of dipoles, is an open problem. Note that for an application of the formalism to mesoscopic dipoles (attributed to nanocrystals) it is more convenient to add the self-energy to $\mathscr{H}_{S}$ as done in (38) because it will depend on relative orientation of dipoles.

Suppose now that the magnetization of the volume unit $\Omega$ obeys the simple law

$$
\mathbf{p}(\mathbf{k})=M_{S} \Omega x_{\Omega} \mathbf{n}(\mathbf{k}),
$$

where $x_{\Omega} \leq 1$. Then we obtain for $k \xi_{m} \gg 1$, using an average of $\delta \mathscr{H}^{\prime}(\mathbf{k})=\delta \mathscr{H}_{J}^{\prime}(\mathbf{k})+\delta \mathscr{H}_{S}^{\prime}(\mathbf{k})$,

$$
\delta \mathscr{H}_{J}^{\prime}(k) / \delta \mathscr{H}_{S}^{\prime}(k) \cong 3\left(k /\left(\kappa_{S} x_{\Omega}\right)\right)^{2} .
$$

Inserting for $\Omega$ the volume of the lattice unit cell requires $x_{\Omega}=1$, and we obtain dominance of the magnetostatic energy for $k<\kappa_{S} / \sqrt{3}$. In case we refer $\Omega$ to the size of a nanocrystal $\left(\Omega_{\mathrm{NC}}\right)$ we have $x_{\Omega}<1$, implying that the magnetization of a nanocrystal in NS material is reduced with respect to a single nanocrystal because (1) nanocrystals do not fit together tightly, and (2) due to form anisotropy and an irregular surface geometry, $N(r)$ in nanocrystals may be weakly nonuniform. For consistency of this model we need $k \leq \pi / \Omega_{\mathrm{NC}}^{1 / 3}$ and for that regime this implies

$$
\max \left[\delta \mathscr{H}_{J}^{\prime}(k) / \delta \mathscr{H}_{S}^{\prime}(k)\right] \cong 3\left(\frac{\pi}{\kappa_{S} \Omega_{\mathrm{NC}}^{1 / 3} x_{\Omega_{\mathrm{NC}}}}\right)^{2} .
$$

Accordingly dominance of magnetostatic energy arises for

$$
k<k_{m} \equiv \min \left[\kappa_{S} x_{\Omega_{\mathrm{NC}}} / \sqrt{3}, \pi / \Omega_{\mathrm{NC}}^{1 / 3}\right] .
$$

The model may further be generalized to the case that the exchange constant $\left(J_{S}\right)$ between nanocrystals is reduced with respect to the bulk $\left(J_{b}\right)$. This implies that the modes satisfying

$$
k_{n} \Omega_{\mathrm{NC}}^{1 / 3} \cong n \pi, \quad n \in \mathbb{Z}
$$

amplify $\delta \mathcal{H}_{S}^{\prime}(k)$ with respect to $\delta \mathscr{H}_{J}^{\prime}(\mathbf{k})$, as follows from (44) with $\kappa_{S}^{-1} \rightarrow \kappa_{S ; k n}^{-1} \equiv \kappa_{S}^{-1}\left(J_{S}\right)<\kappa_{S}^{-1}\left(J_{b}\right)$. Note that the number of modes in (47) for $k_{n} \leq \pi / a$ is equal to the number of magnetic degrees of freedom of a single nanocrystal, whereas the modes $k<k_{m}$ describe the collective magnetic degrees of freedom of the NS ferromagnet.

Let us point out that the ansatz (43) for $\Omega \cong \Omega_{\mathrm{NC}}$ and $x_{\Omega}<1$ is not very realistic because in that domain it should be replaced by a nonlinear law

$$
\mathbf{p}\left(\mathbf{r}_{i}\right)=\mathbf{p}\left(\mathbf{r}_{i}, \mathbf{N}\left(\mathbf{r}_{i}\right)\right),
$$

which takes account of the fact that the magnetic surface charge may vary strongly with the orientation of $N(r)$. This leads naturally to a trapping of the order parameter caused by the nontrivial structure of the labyrinthine domain $\mathcal{L}$, and it can be postulated safely that the majority of the modes $k<k_{m}$ is frozen out at low temperatures. This effect is well known in the micromagnetics of small particles and is a consequence of form anisotropy. ${ }^{27}$
The physical significance of the model presented is that in an ideal Heisenberg magnet the magnetostatic energy modifies the dispersion and structure of magnons strongly in the domain $k \leq \kappa_{S} / \sqrt{3}$. In contrast to that we have in a NS Heisenberg magnet additional degrees of freedom due to magnetically charged internal surfaces implying an additional modification for $k \leq k_{m}<\kappa_{S} / \sqrt{3}$. A computation of the excitational modes is based on (9) and has been done by Cohen and Keffer ${ }^{29}$ for the case of a uniformly magnetized Heisenberg magnet (see also Ref. 30). Due to the constraint $N^{2}(\mathbf{r})=1$, small amplitude excitations $[\delta \mathbf{N}(\mathbf{r})]$ are transversally polarized to $\mathbf{N}(\mathbf{r})$. A computation of the excitational modes for our model of a NS Heisenberg magnet, however, is more involved because (43) has to be replaced by a nonlinear law $\left(43^{\prime}\right)$ and, therefore, will not be presented here. Ultimately, however, one will have to apply the methods developed in Ref. 28 to find the magnetic ground-state configurations of NS ferromagnets in the presence of the law $\left(43^{\prime}\right)$, and the method developed in Ref. 29 to verify its quantummechanical stability. Due to the reduced symmetry of a stochastic dense packing array of dipoles this calls for the diagonalization of large matrices corresponding to the quadratic form defined by (38) and $\left(43^{\prime}\right)$. Qualitatively the modes for $k<k_{m}$ may be compared to the surface modes (Walker modes, see, e.g., Ref. 30) and rely essentially on the structure of the labyrinthine domain.

In order to understand the physical significance of magnetostatic modes we associate them with a characteristic temperature $T_{S}$ as follows. Using the replacement $k^{2} \rightarrow 2(1-\cos k a) / a^{2}$ in $\delta \mathcal{H}_{J}^{\prime}(k)$ we obtain a rough estimate for the ratio

$$
\delta \mathcal{H}^{\prime}\left(\kappa_{S} / \sqrt{3}\right) / \delta \mathcal{H}_{J}^{\prime}(\pi / a) \cong \frac{1}{6}\left(a \kappa_{S}\right)^{2} \cong T_{S} / T_{C},
$$

where $T_{C}$ is the Curie temperature. This implies for $\mathrm{Fe}$ and $\mathrm{Ni}, T_{S} / T_{C} \cong \frac{1}{6} \times 10^{-2}$ and $\frac{1}{3} \times 10^{-3}$, respectively. In a similar fashion, one can associate a characteristic temperature $T_{m}$ with the modes $k^{\prime} \leq k_{m}$, in case the linear law (43) applies, for which one obtains $\left(T_{m} / T_{S}\right) \cong(1 / 3)\left(\kappa_{S}^{-2} / \Omega_{\mathrm{NC}}^{1 / 3}\right)^{2} \ll 1$. From this follows that the characteristic temperatures $T_{S}$ and $T_{m}$ are extremely small, and therefore the modes associated with them should be considerably populated thermally at $T \gg T_{S}$, which is still of the order of $1 \mathrm{~K}$ for Ni. This implies that nonlinearities of these modes play a major role. Because the $T$ dependence of the saturation magnetization $M_{S}(T)$ depends sensitively on the dispersion of the magnon excitations [e.g., the $T^{3 / 2}$ law requires $\omega(k) \sim k^{2}$ for $k \rightarrow 0$ ], it can be expected that at $T<T_{m}$ and $T<T_{S}$, this law will change with respect to $T>T_{S}$.

For the case that a nonlinear version $\left(43^{\prime}\right)$ of the law (43) applies, implying the existence of frozen-in magnetostatic modes for $k<k_{m}, T_{m}$ will increase yielding $T_{m} \gg T_{S}$. Empirically this follows at least for $\mathrm{Ni}$ from the observation that $\partial M_{S}(T) / \partial T$ for NS and PC material has the same low-temperature behavior, ${ }^{2,3}$ and the relative capacity of the magnetic degrees of freedom for $k<k_{m}$ is negligible for $T_{m}>T_{S}$. However, in case $T_{m} \gtrsim T_{S}$ holds, a modification of that law is expected. The qualitative estimate $T_{m} \gg T_{S}$ in NS magnets is based 
on the assumption that their magnetic ground state is nonuniform on the length scale $d_{\mathrm{NC}}$. In particular locally (the neighborhood of adjacent nanocrystals) the exchange and stray field energy will depend on the relative orientation of magnetization in adjacent nanocrystals. The point now is that releasing the trapped in modes requires a screening of magnetic charge and in addition a weakening of the effective exchange coupling between adjacent nanocrystals. The first mechanism may be provided by the dipolar modes $k<\kappa_{S} / \sqrt{3}$, once being strongly populated thermally, i.e., $T_{m} \gg T_{S}$ is required. The second mechanism sets in once the regime of modes $k \gtrsim \pi / \bar{d}_{\mathrm{NC}}>\kappa_{S} / \sqrt{3}$ propagating in the labyrinth $\mathcal{L}$ becomes thermally populated.

On the other hand, the temperature interval $\left[T_{S}, T_{m}\right]$ may also be associated with the strong initial decrease of the coercive force in Ni (see Sec. VI), where no effect on $\partial M_{S}(T) / \partial T$ is observed, implying $T_{m} \gg T_{S}$. From this one may conclude that the modes $k<k_{m}$ have the character of nontopological solitons, whose excitation is provided by thermal energy, and where $T_{m}$ marks roughly a transition from a frozen-in state into a plasma state. In this scenario topological defects, nontopological solitons, and magnetostatic modes form a hierarchy of excitations in a NS ferromagnet. In this sense the structure of the disclination network is strongly dependent on the properties of nontopological solitons. Once the latter form a plasma state they will provide for a partial screening of interactions within the network of disclinations leading to a softening of the latter and to a considerable reduction of the coercive force. In addition, pinning of the disclination core may successively be reduced in the interval $\left[T_{S}, T_{m}\right]$ and suppressed for $T \geq T_{m}$. This may also be accompanied by a change of the law $d Q_{H_{H}} / d H_{c}$ at $T_{m}$ as indicated by (34) in Sec. III.

Let us briefly consider the significance of the collective degrees of freedom with $k<\pi / \Omega_{\mathrm{NC}}^{1 / 3}$ upon approaching $T_{C}$. Because in NS magnets we will have $J_{s}<J_{b}$ it is obvious that these modes also play a major role for the longitudinal order parameter excitations. It follows then from (46) that for $J_{S}=0$ [implying $\kappa_{S}^{1 / 2}\left(J_{s}=0\right)=0$ ] all collective modes are dominated by magnetostatic energy, whereas for $J_{s}>0$ only those satisfying $k<k_{m}$ $=\kappa_{S} x_{\Omega_{\mathrm{NC}}} / \sqrt{3}$. From this follows that upon approaching $T_{C}$ critical fluctuations eventually become dominated by long-range interaction implying mean-field behavior. The consequence of that for NS ferromagnets will be indicated in Sec. V.

Eventually it is pointed out that the elastic relaxation time $\tau_{e}(k)$ of modes obeying $k>\kappa_{S} / \sqrt{3}$ is geometrically confined by the linear dimension $d_{\mathrm{NC}}$ of nanocrystals in the skeleton. A simple estimate of $\tau_{e}(k)$ is obtained as follows. Using the dispersion $\omega \cong c k^{2}$ for $k>\kappa_{S} / \sqrt{3}$ and the group velocity $v_{\mathrm{g}} \cong 2 c k$ one obtains $\tau_{e} \gtrsim d_{\mathrm{NC}} / 2 c k$. Because $T>T_{S}$ represents the low-temperature regime (according to the estimates given earlier), the physical consequences of that for the behavior of the temperature laws for $M_{S}(T)$ and the specific heat due to magnons, may be considerable. This problem will be studied elsewhere.

\section{MICROMAGNETIC APPLICATIONS TO NS FERROMAGNETS}

In order to apply the present theory to a NS ferromagnet the internal magnetic surface structure at $\partial_{i} \mathcal{M}$ and $\partial_{i} \mathcal{\rho}$ (see Fig. 1) must be accounted for, which implies in fact that such a system is not an ideal Heisenberg magnet. For the sake of simplicity we assume in the following first $\partial_{i} M=\emptyset$. Because the magnetic properties of nanocrystals and CS material differ, e.g., $M_{S}$ in the latter is reduced by about $50 \%$ (Refs. 1-3) due to a reduction of density, $\partial_{i} \mathcal{S}$ is associated with a magnetic charge density, which extends to a depth of about $\frac{1}{2} \kappa_{S}^{-1}$ to either side of $\partial_{i} \mathcal{S}$. However, this will not be associated with discontinuities of the $\mathbf{N}$ field, provided the exchange constant $J(\mathbf{r})$ and $M_{S}(\mathbf{r}, T)$ do not vanish along $\partial_{i} \mathcal{\rho}$. In that case the representation $\left(16^{\prime}\right)$ of Hopf's invariant (15) or (16) is still valid, and so is its change in time given by (21) or $\left(21^{\prime}\right)$. It is the presence of the charged surfaces $\partial_{i} \mathcal{S}$, which essentially implies that NS ferromagnets differ from ideal Heisenberg magnets. This holds, of course, only modulo dissipative couplings and quantum excitations. An example of the latter system may be europium sulfide (EuS), which has a small crystalline anisotropy constant and undergoes a classical Curie transition at $T_{C}=16.57 \mathrm{~K}^{31}$ Note that the boundaries $\partial_{i} \rho$ have different magnetic properties with respect to those of $\partial_{e} \mathcal{M}$ (in the absence of pores extending to the visible surface of the sample $M$ ) because $\partial_{i} \rho$ will be homeomorphic to a rather intricate two-manifold embedded in $\mathbb{R}^{3}$. The boundary conditions to which $\mathbf{M}(T, \mathbf{r})$ is subject at $\partial_{e} \mathcal{M}$ and indicated earlier will therefore not apply at $\partial_{i} \rho$ due to the fact that $d_{\mathrm{NC}} \kappa_{S} \gg 1$ is not realized, and, in addition, due to the possibility of screening of magnetic charge for $\kappa_{S}^{-1} \sim \bar{d}_{\mathrm{NC}}$.

\section{A. Model of the saturation magnetization}

Let us point out that the saturation magnetization $\mathfrak{M}_{\mathrm{NS}}(0)$ (in units of emu/gr) in NS Ni was determined in Ref. 2 in applied fields of $H \lesssim 5.5 \mathrm{~T}$ by extrapolation to $H=0$ and $T=0$, yielding $50 \mathrm{emu} / \mathrm{gr}$ in contrast to 59 $\mathrm{emu} / \mathrm{gr}$ in crystalline Ni. Because the applied field $H \cong 5.5 \mathrm{~T}$ corresponds in $\mathrm{Ni}$ to a characteristic length $\kappa_{H}^{-1} \cong 2.55 \mathrm{~nm}$, we have now $\kappa_{S}^{-1} \lesssim 3 \kappa_{H}^{-1}$, implying that the magnetic moments should be well aligned and extrapolation to $\mathfrak{M}_{\mathrm{NS}}(0)$ well defined. In that case we can compute an upper bound of $\mathfrak{M}_{\mathrm{NS}}(0)$ using only the second term of (5b) with $\partial^{\prime} \mathcal{M}=\partial_{e} \mathcal{M}+\partial_{i} \mathcal{S}+\partial_{i} \mathcal{L}$. Setting $\rho_{c}, \rho_{\mathrm{NS}}$, and $\rho_{\mathrm{CS}}$ for the densities of the crystalline, NS, and CS material, respectively, and in an analogous fashion $M_{n}(0), M_{\mathrm{NS}}(0)$, and $M_{\mathrm{CS}}(0)$ for the saturation magnetizations (volume densities) we obtain

$\rho_{\mathrm{NS}}=x \rho_{c}+y \rho_{\mathrm{CS}}, \quad M_{\mathrm{NS}} \leq x M_{c}(0)+y M_{\mathrm{CS}}(0), \quad 1=x+y$.

Here $x$ and $y$ represent the volume fractions of crystalline and CS material, respectively. Setting $\rho_{\mathrm{NS}}=\alpha \rho_{c}$, $\rho_{\mathrm{CS}}=\beta \rho_{c}$, where $1>\alpha>\beta$, we obtain 
$\mathfrak{M}_{\mathrm{NS}}(0) \leq\left(\frac{1-\beta / \alpha}{1-\beta}\right) \mathfrak{M}_{c}(0)+\left(\frac{1 / \alpha-1}{1 / \beta-1}\right) \mathfrak{M}_{\mathrm{CS}}(0)$,

where $\mathfrak{M}_{\mathrm{NS}}(0) \equiv M_{\mathrm{NS}}(0) / \rho_{\mathrm{NS}}$, etc. Setting $\alpha=0.8$ and $\mathfrak{M}_{\mathrm{NS}} \cong 0.84 \mathfrak{M}_{c}$ for NS Ni (Refs. 2 and 3 ) and $\beta \cong 0.5$ (Ref. 1) we obtain

$$
\mathfrak{M}_{\mathrm{CS}}(0) \geq 0.36 \mathfrak{M}_{c}(0) \text {. }
$$

Observe now that the magnetic moments of $\mathrm{Fe}$, $\mathrm{Co}$, and $\mathrm{Ni}$ depend on the local electronic environment, implying that the moments increase with decreasing coordination number $z$ of the lattice, and increasing interatomic distance. ${ }^{32}$ In addition there is a dependence of the exchange constant $J$ on the interatomic distance, qualitatively described by the Bethe-Slater curve. ${ }^{27}$ Under the preliminary assumption that $J$ changes little in the CS material it follows from this that $\mathfrak{M}_{\mathrm{CS}}(0)$ should increase with respect to $\mathfrak{M}_{c}(0)$, i.e.,

$$
\mathfrak{M}_{\mathrm{CS}}(0)>\mathfrak{M}_{c}(0),
$$

and therefore the inequality given above is rather inaccurate for the equality sign. One may conclude now that $J(\mathbf{r})$ entering (6) is strongly reduced in CS material due to a reduction of the coordination number $z$ (as follows from $\beta \cong 0.5)$, implying that the susceptibility $\chi_{\mathrm{CS}}(H, T)$ of the CS material at $H \leq 5.5 \mathrm{~T}$ is enhanced with respect to the crystalline material. As a matter of fact, one has to assume that $\chi_{\mathrm{Cs}}(H, T)$ is the susceptibility of a paramagnet with a magnetization density $\left[\mathfrak{M}_{\mathrm{CS}}(0)-0.36 \mathfrak{M}_{c}(0)\right]$ $\geq 0.64 \mathfrak{M}_{c}(0)$ in the applied field $H$, and which vanishes upon extrapolation to $H=0$, in order that the quality sign in the relation (51) given above holds.

The proper interpretation of our result is presumably that despite the strong applied fields $\left(\kappa_{S}^{-1} / \kappa_{H}^{-1} \lesssim 3\right)$ used to measure the saturation magnetization, ${ }^{2,3}$ the magnetic susceptibility $\chi_{\mathrm{NS}}(H, T)$ contains considerable contributions from magnetostatic sources in the sample. This would imply then that an extrapolation to zero magnetic field yields a magnetically nonuniform ground state, and the upper bound of $\mathfrak{M}_{\mathrm{NS}}(0)$ computed above strongly overestimates the experimental results. Note that this is certainly the case in the magnetic field domain $\kappa_{S}^{-1} / \kappa_{H}^{-1} \cong 1$ because after turning off the magnetic field the system should relax into a nonuniform configuration, e.g., into that of Fig. 2(a). Consequently $\chi_{\mathrm{NS}}(H, T)$ will also extrapolate into a configuration close to that and not to the uniform magnetic configuration assumed for the computation of the upper bound of $\mathfrak{M}_{\mathrm{NS}}(0)$. Besides the interpretation given above a reduction of the upper bound may also be explained by the presence of trapped magnetic flux in the system, which in strong applied fields $\left(\kappa_{S}^{-1} / \kappa_{H}^{-1} \lesssim 3\right)$ is less likely, observing that for a Néel wall we have $\left(\pi \kappa_{S}^{-1} / \kappa_{H}^{-1}\right) \cong 10$ for $H \cong 5.5 \mathrm{~T}$.

\section{B. Magnetization configuration close to planar surface}

An important problem is to find out how many openended disclinations (piercing through $\partial_{e} \mathcal{M}$ ), closed loops of disclinations and entangled disclinations does the sys- tem contain. This will certainly depend on the geometry of $\partial_{e} \mathcal{M}$ and its area, on the specific process of generation of the magnetic state, and the topological stability of the entangled structures. In particular due to $\pi \kappa_{S}^{-1}>\bar{d}_{\mathrm{NC}}$ and $\pi \kappa_{S}^{-1} \sim O\left(d_{\mathrm{NC}}\right)$ in NS ferromagnets, global magnetization structures are constrained by optimization of local magnetostatic and exchange energy being compatible with the geometry of the external surface. We postulate therefore that in the generic situation the system will contain many open-ended disclinations with their number being proportional to the surface area, and that they should be visible at the surface of the sample using suitable methods of observation. A model distribution for a planar surface $\partial_{e} \mathcal{M}$ with normal $\mathbf{n}=(0,0,1)$, may be given in polar coordinates $(\gamma, \beta)$ and $\mathbf{N}$ $=(\sin \beta \cos \gamma, \sin \beta \sin \gamma, \cos \beta)$ in the form

$$
\begin{aligned}
& \gamma(\mathbf{r})=\sum_{i=1}^{N} s_{i} \arctan \left(\frac{Y-y_{i}}{X-x_{i}}\right)^{s_{i}}+\frac{\pi}{2} g\left(\mathbf{r},\left\{r_{i}, s_{i}\right\}\right), \\
& s_{i} \in \mathbb{Z}, \mathbf{r} \in \partial_{e} \mathcal{M}, \\
& \beta(\mathbf{r})=\frac{\pi}{2}\left[1-\sum_{i=1}^{N} s_{i}^{\prime} \exp \left(-\left|\mathbf{R}-\mathbf{r}_{i}\right| \kappa_{S}\right)\right], \quad s_{i}^{\prime}= \pm 1 .
\end{aligned}
$$

Here $\left\{\mathbf{r}_{i}\right\}$ are the locations of singularities, and $\mathbf{R}=(X(\mathbf{r}), Y(\mathbf{r}))$ is a smooth function of $\mathbf{r}$, satisfying $\mathbf{R}\left(\mathbf{r}_{i}\right)=\mathbf{r}_{i}, i=1, \ldots, N$, whose form should be determined by means of the variational principle $\delta \mathscr{H}=0$. Furthermore, $g$ in (52) is a smooth function, which should be chosen such, that for $\left|\mathbf{r}-\mathbf{r}_{i}\right| \ll<\left|\mathbf{r}_{i}-\mathbf{r}_{j}\right|$ and all $r_{j} \neq r_{i}$, we get

$$
\gamma(\mathbf{r}) \cong s_{i} \arctan \left[\left(y-y_{i}\right) /\left(x-x_{i}\right)\right]^{s_{i}}+(\pi / 2) \delta_{s_{i}, 1}
$$

for $s_{i}= \pm 1$, implying $\boldsymbol{\nabla} \cdot \mathbf{N} \cong 0$, i.e., avoidance of magnetic volume charge in the neighborhood of the set $\left\{\mathbf{r}_{i}\right\}$. In (52) $\left|\mathbf{r}_{i}-\mathbf{r}_{j}\right| \kappa_{S} \gg 1$ is assumed, and $s_{i}^{\prime}$ represents the polarization in $\hat{z}$ direction of the core of the $i$ th disclination, whereas $s_{i}$ represents its vorticity. In order to obtain a finite energy $\mathscr{H}$ for an infinitely extended system $\Sigma_{i}^{N} s_{i}=0$ is required. This is illustrated in Fig. 4 for $N=4$. For a curved surface $\partial_{e} M,(52)$ has to be subject to a mapping

$$
(\gamma(\mathbf{r}), \beta(\mathbf{r})) \rightarrow\left(\gamma^{\prime}\left(\mathbf{r}^{\prime}\right), \beta^{\prime}\left(\mathbf{r}^{\prime}\right)\right)=\left(\gamma\left(\mathbf{r}\left(\mathbf{r}^{\prime}\right)\right), \beta\left(\mathbf{r}\left(\mathbf{r}^{\prime}\right)\right)\right)
$$

with $r^{\prime} \in \partial_{e} \mathcal{M}$, and $\mathbf{N}$ is defined with respect to the normal $\mathbf{n}^{\prime}$ to the tangent space $T\left(\partial_{e}, \mathcal{M}\right)$ at $\mathbf{r}^{\prime}$.

A simple continuation of (52) into the bulk is obtained via the replacement of $\beta(r)$ in (52) by

$$
\begin{aligned}
\beta(\mathbf{r}, z)= & \frac{\pi}{2} f\left(z \kappa_{S}\right)\left[1-\sum_{i=1}^{N} \frac{\left(1+s_{i}^{\prime}\right)}{2} e^{-\left|\mathbf{R}-\mathbf{r}_{i}\right| \kappa_{S}}\right] \\
& +\frac{\pi}{2} \sum_{i=1}^{N} \frac{\left(1-s_{i}^{\prime}\right)}{2} e^{-\left|\mathbf{R}-\mathbf{r}_{i}\right| \kappa_{S}},
\end{aligned}
$$

where $f\left(z \kappa_{S}\right)$ is subject to the boundary conditions $f(0)=f\left(d \kappa_{S}\right)=1 \quad(d$ is the thickness of the specimen $)$ and determined by the variational principle $\delta \mathscr{H}=0$. Entanglements of disclinations are generated via the mappings $r_{i} \rightarrow r_{i}(z)$ for $i=1, \ldots, N$. The magnetic surface 
singularities move, upon change of the applied magnetic field, similarly as do Bloch walls, i.e, $\mathbf{r}_{i} \rightarrow \mathbf{r}_{i}(t)$ and $\mathbf{R} \rightarrow \mathbf{R}(\mathbf{r}, t)$. Observe that for $\left|\mathbf{R}-\mathbf{r}_{i}\right|>\kappa_{S}^{-1}$ we have $\beta(\mathbf{r}) \cong \pi / 2$ and the magnetization distribution given above corresponds to that of an $X Y$ model. Alternatively we have for $\left|\mathbf{R}-\mathbf{r}_{i}\right|<\kappa_{S}^{-1}, \beta(\mathbf{r}) \neq \pi / 2$ and escape of the magnetization into the third direction ( $\hat{z}$ coordinate) eliminating the vortex-type singularities at $\mathbf{r}=\left\{\mathbf{r}_{i}\right\}$ occurring in an $X Y$ model. This implies that the magnetic surface charge is not concentrated at $\mathbf{r}=\mathbf{r}_{i}$ but in a diskshaped area of radius $\pi \kappa_{S}^{-1}$ (see Sec. II). In addition (52) implies for $g \equiv 1$ the presence of a magnetic charge density $\rho_{m}=-\nabla \cdot M$, which behaves for $\mathbf{R}(\mathbf{r}) \cong \mathbf{r}$, and $\left|\mathbf{r}-\mathbf{r}_{i}\right| \ll\left|\mathbf{r}_{i}-\mathbf{r}_{j}\right|$ for all $\left\{\mathbf{r}_{j} \neq \mathbf{r}_{i}\right\}$ approximately as $\rho_{m}(r) \sim s_{i} /\left|\mathbf{r}-\mathbf{r}_{i}\right|$ for $\left|\mathbf{r}-\mathbf{r}_{i}\right| \kappa_{S} \gg 1$. The fact that the order-parameter field is charged cannot be avoided in this approach. A change of polarization of the core of a disclination $\left(s_{i}^{\prime} \rightarrow-s_{i}^{\prime}\right)$, may be described using $\left(52^{\prime}\right)$ with $s_{i}^{\prime} \rightarrow s_{i}^{\prime}(t)$. For $s_{i}^{\prime}(t)=0$, this yields a core singularity, which is unavoidable in such a magnetization reversal, and corresponds to the topological singularities studied in Sec. III.

In the simplest case one may set in (52) and (52') the functions $f, g \equiv 1, \mathbf{R}(\mathbf{r}) \cong \mathbf{r}$, and $d \ll\left|\mathbf{r}_{i}-\mathbf{r}_{j}\right|$ for $i \neq j$. Then one obtains for the energy (4) of the magnetization structure (52) for a system of linear dimension $L$

$$
\begin{aligned}
\mathcal{H} \cong & -J d \alpha \sum_{i \neq j}^{N} s_{i} s_{j} \ln \left(\left|\mathbf{r}_{i}-\mathbf{r}_{j}\right| \kappa_{S}\right)-J\left(d \kappa_{S}\right) \sum_{i, j}^{N} s_{i} s_{j}\left[\beta+\gamma \ln \left(L /\left|\mathbf{r}_{i}-\mathbf{r}_{j}\right|\right)\right]\left|\mathbf{r}_{i}-\mathbf{r}_{j}\right| \\
& +J d^{2} \delta \sum_{i \neq j}^{N} \frac{s_{i} s_{j}}{\left|\mathbf{r}_{i}-\mathbf{r}_{j}\right|}+J \kappa_{s}^{-2} \epsilon \sum_{\sigma=0,1} \sum_{i \neq(1-\sigma) j}^{N} \frac{s_{i}^{\prime} s_{j}^{\prime}(1-2 \sigma)}{\left(\left|\mathbf{r}_{i}-\mathbf{r}_{j}\right|^{2}+\sigma d^{2}\right)^{1 / 2}}+J d \lambda_{1} \sum_{i=1}^{N} s_{i}^{2}+J \kappa_{S}^{-1} \lambda_{2} \sum_{i=1}^{N} s_{i}^{\prime 2}-\kappa_{S}^{-2} d H \lambda_{3} \sum_{i=1}^{N} s_{i}^{\prime} .
\end{aligned}
$$

Here $\alpha$ to $\epsilon$, and $\lambda_{1}$ to $\lambda_{3}$ are positive $O(1)$ constants. The first term in (53) refers to the exchange energy, the second and third terms to the magnetostatic energy of bulk magnetic charge, and the fourth term represents the interaction energy of magnetic surface charge. The last three terms refer to the self-energy of the core of disclinations and to their magnetostatic energy in the applied field $H$. The appearance of $L$ in the second term of (53) signals, that lateral boundary conditions will play a role except if $\gamma=0$ or $\sum_{i, j} s_{i} s_{j}\left|\mathbf{r}_{i}-\mathbf{r}_{j}\right|=0$. If the latter condition for a regular lattice arrangement of disclinations can be satisfied is doubtful. Because for that one may require $\sum_{i} s_{i} \mathbf{r}_{i}=0$, implying $\sum_{i, j} s_{i} s_{j}\left|\mathbf{r}_{i}-\mathbf{r}_{j}\right|^{2}=0$ and from which follows $\sum_{i, j} s_{i} s_{j}\left|\mathbf{r}_{i}-\mathbf{r}_{j}\right| \neq 0$. The energy $\mathscr{H}$ should be compared to $\mathscr{H}^{0}=\left(2 \pi M_{S}-H\right) M_{S} V$, where $V$ is the volume of the specimen, and which applies to a "normal" magnetization. Only for $\mathscr{H}-\mathscr{H}^{\circ}<0$, and $\mathscr{H} \sim N$ such a model makes sense, but due to the difficulties produced by the second term of (53) the problem has not been resolved so far.

The structures described by (52) and (52') are, in fact, not general enough because they do not describe disclination cores being oriented parallel to the surface, e.g., in the form of rings. An ansatz describing that situation is given by (60), but is much more difficult to handle. Consider, e.g., a ring-shaped disclination between two planar surfaces. Then the field $\mathbf{N}(\mathbf{r})$ forms a toroidal configuration, and for $\mathbf{N}(\mathbf{r})$ at $\mathbf{r} \in \partial_{e} \mathcal{M}$ being tangential to the external surface, a straight disclination passes through the center of the ring leaving magnetic poles at points where it pierces through the surface. The situation may be compared to that arising in a RayleighBénard system, ${ }^{33}$ when $\mathbf{N}(\mathbf{r})$ is mapped onto the field lines of the flow of a single convection cell of polygonal shape. Extension of the model distribution to a (hexagonal) lattice of (polygonal) rings, however, makes the anal- ogy even closer. It is also suggestive that some of the methods, in particular the stability analysis, developed in this field may also be applied successfully to the present problem. Furthermore, it is possible that some of the regular features of domain structures observed in classical ferromagnets are also reproduced in NS material. In this context we like to point out that the imprint of disclinations, in the examples considered above, at a surface oriented perpendicular to their orientation will be a texture reflecting the 1D nature of their cores. For the case that the polarization $\left\{s_{i}^{\prime}\right\}$ of cores alternates in space the formation of domain structures at the surface is conceivable. Furthermore, for very thin films and $\kappa_{S}^{-1} \ll \kappa_{H}^{-1}$,

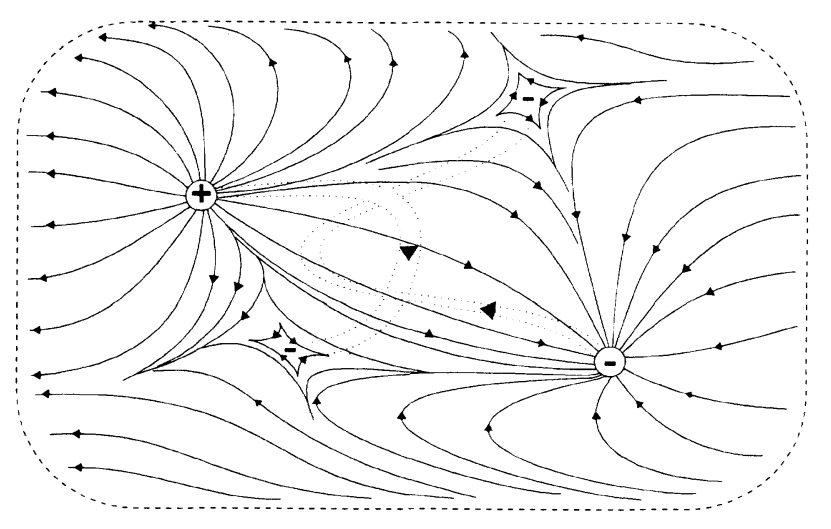

FIG. 4. Example of quadrupolar order parameter field on a flat surface and on the $S^{2}$ surface of a solid ball $\mathscr{B}$. In the latter case points along the dashed drawn circumference are identified to a point. The figure may also be viewed as stereographic projection of $S^{2}$ on Euclidean plane with the dashed drawn circle moved to infinity and corresponding, e.g., to the north pole of $S^{2}$. Disclination lines are drawn dashed and connect positive and negative magnetic poles through bulk of $\mathcal{B}$. 
demagnetization of planar surface may be accomplished over a domain structure.

\section{Asymptotic equilibrium configuration}

Another interesting problem is the computation of the internal energy $\mathscr{H}(H, M, T)$ along some $(M, H)$-hysteresis curve. From a topological point of view this problem may be attacked by the methods developed recently by Moffatt. ${ }^{34}$ This author studies the asymptotic equilibrium configurations of knots of any complexity, which he associates with positive numbers $m_{i} \geq m_{0}>0$, $\left(m_{i+1} \geq m_{i}\right)_{i=1,2,3, \ldots}$ and which he calls the spectrum of a knot with $m_{0}$ referring to its ground state. The equilibrium configurations are reached via an incompressible flow field $\{\mathbf{v}\}$, and in the present context this may be replaced by the corresponding volume preserving diffeomorphism of the $\mathbf{N}(\mathbf{r})$ field. See the remark made in Sec. II in connection with the Möbius transformation (19) of $S^{2}$. Relaxation in the system comes to a halt, once different parts of flux tubes come into contact and provide topological barriers to stabilize $\mathscr{H}$. Rewriting (5a) and (7) in the form

$$
\begin{aligned}
\mathscr{H}_{S}+\mathscr{H}_{H}= & -\frac{1}{8 \pi} \int_{\mathcal{M}} d^{3} r \mathbf{B}^{2}-\frac{1}{4 \pi} \int_{\mathcal{M}} d^{3} r \mathbf{H} \cdot \mathbf{B} \\
& +2 \pi \int_{\mathcal{M}} d^{3} r M^{2}(\mathbf{r})
\end{aligned}
$$

we obtain for (4) in a tentative translation of Moffatt's ${ }^{34}$ approach to the present problem

$$
\begin{aligned}
& \mathscr{H}(H, M, T)-2 \pi \int_{\mathcal{M}} d^{3} r \mathbf{M}^{2}(\mathbf{r}) \cong \sum_{j}\left\{-\frac{1}{8 \pi} m_{i(j)} \Phi_{i(j)} / V_{i(j)}^{1 / 3}+\frac{1}{4} m_{i(j)}^{\prime}\left(\kappa_{S} V_{i(j)}^{1 / 3}\right)^{-2} \Phi_{i(j)}^{\prime 2} / V_{i(j)}^{1 / 3}\right\} \\
&-\left(\frac{\kappa_{H}}{\kappa_{S}}\right]^{2} M_{S} \sum_{j}^{\prime} \Phi_{i(j)} \int_{\mathbf{r}_{j}^{-}}^{\mathbf{r}_{j}^{+}} d \mathbf{r} \cdot \mathbf{h}_{0} \\
& V=\sum_{j} V_{i(j)} .
\end{aligned}
$$

Here it is assumed that magnetic configurations in a volume $V$ of $\mathcal{M}$ can be divided into knotted flux tubes (labeled by $j$ ) along which the magnetic flux $\Phi_{i(j)}=\int_{A_{j}} d^{2} r \cdot \mathbf{B}(\mathbf{r})$ is conserved, i.e., the flux tube is bounded by a magnetic surface. The volume of the $j$ th flux tube is $V_{i(j)}$ and its cross section and linear extension is $A_{i(j)} \sim V_{i(j)}^{2 / 3}, L_{i(j)} \sim V_{i(j)}^{1 / 3}$, respectively. Using

$$
\Phi_{i(j)}^{\prime} \simeq 4 \pi \int_{A_{i(j)}} d^{2} \mathbf{r} \cdot \mathbf{M}(\mathbf{r})
$$

one obtains (55a), where $m_{i(j)}$ is the $i$ th number of the $j$ th knot and $m_{i(j)}^{\prime} \sim m_{i(j)}, \mathbf{H}=H \mathrm{~h}_{0}$. For a set of linked flux tubes of different strengths the spectral numbers in (55a) are not all independent. The terms on the right-hand side of (55a) refer to stray field, exchange, and magnetostatic energy, respectively. The sum in the last term of (55a) runs only over open-ended flux tubes extending from $\mathbf{r}_{j}^{-}$ to $\mathbf{r}_{j}^{+}$, whereas in the first term it runs over open-ended and closed-flux tubes as well. Although Moffatt ${ }^{34}$ does not give a prescription how to compute the topological invariants $\left\{m_{i}\right\}$, it can be assumed that they also do not vanish for open-ended and braided flux tubes using the concepts explained below (20). Equations (55) apply for $\left(\kappa_{H} / \kappa_{S}\right)^{2} \ll<1$.

Within our theory the motion along a $(M, H)$ hysteresis curve can be brought in connection with topological transmutations of knots and links and a reversible change of the magnetization. On account of that, it cannot be a differentiable curve but may have a fractal character. Mobility of topological defects may be reduced if they are located at pinning centers of the surface and the bulk, e.g., pores, which confine the tubelike core of a disclination. Excitations of the order parameter in that case may be described by subjecting it to local $S O(2)$ transformations at $\partial_{e} \mathcal{M}$ and suitable extensions into the bulk. In ad- dition pinning may be realized over the form of $\mathbf{R}(\mathbf{r}, t)$ in (52). Depending on the size $\left(d_{p}\right)$ of a pore the singularity associated to (13) will submerge below the visible surface of the sample and in case it extends all through $M$ in a simple fashion we have, e.g., $\mathcal{M} \cong \mathscr{B}$ - pore $\cong$ solid 2 torus, where $\mathscr{B}$ is the solid ball, it may disappear. However, as long as $d_{p} \lesssim \pi \kappa_{S}^{-1}$ it should still be visible at the surface.

\section{Surface coated (NiO) NS Ni}

Suppose now that the system contains internal surfaces, i.e., $\partial_{i} M \neq \emptyset$. This situation will apply in the presence of holes and pores in the NS material and implies that magnetic surface charge has to be taken account of at $\partial_{i} \mathcal{M}, \partial_{i} \mathcal{S}$, and $\partial_{e} \mathcal{M}$. The simplest situation presumably arises for $\partial_{i} M=\partial_{i} \mathcal{\rho}$ and for a model where the nanocrystals of $\mathcal{S}$ are not joined together by magnetic grain boundaries but nonmagnetic material. This case applies roughly to $\mathrm{NS} \mathrm{Ni,} \mathrm{where} \mathrm{nanometer-sized} \mathrm{crystals}$ are surface coated with $\mathrm{NiO} .^{2,3}$ Because $\mathrm{NiO}$ is antiferromagnetic $\left(T_{N} \sim 520 \mathrm{~K}\right)$ and insulating it will disrupt the exchange coupling along $\partial_{i} \mathcal{S}^{\prime}$, where $\mathcal{S}^{\prime}$ is the magnetic skeleton indicated in Fig. 5 forming a subskeleton of the mechanical skeleton $\mathcal{S}$. Similarly $\mathcal{M}^{\prime} \subset \mathcal{M}$ represents the magnetic part of the sample. The hypothetical structure indicated in Fig. 5 is supposed to arise during the formation process of the NS material, where it must be assumed that individual nanocrystals are subject to severe plastic deformation, changing the topology of their coats. For instance it is possible that $\mathcal{\rho}^{\prime}$ forms an infinitely extended cluster, which may be probed via the electrical properties of the NS material. In any case the significance of the exchange constant $J$ for such NS material is strongly reduced, whereas the importance of crystalline anisotropy and particularly of stray fields has 


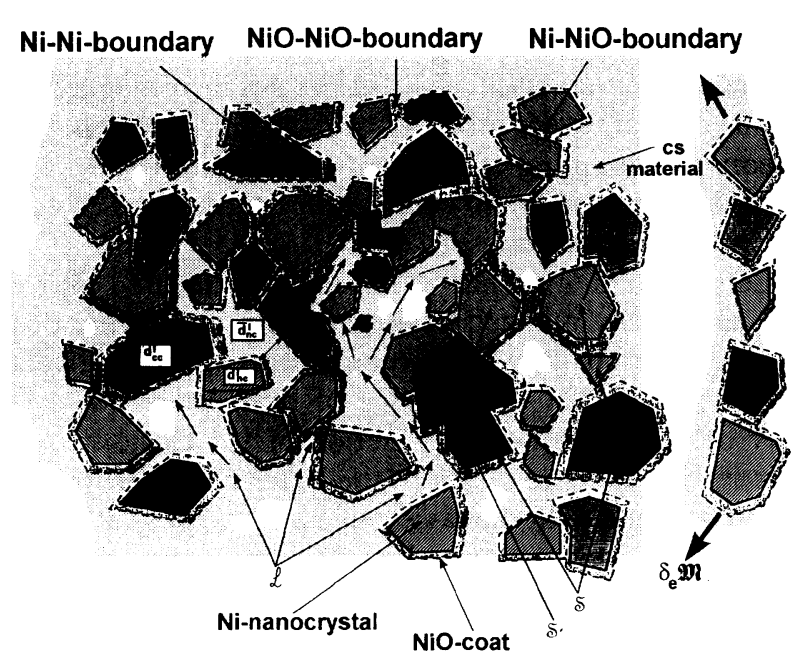

FIG. 5. Schematic illustration of a two-dimensional cross section of a NS material, whose building blocks are Ni nanocrystals surface coated with NiO. The coats of nanocrystals are indicated by dashed drawn lines. The labyrinth $\mathcal{L}$ contains CS material composed mainly of $\mathrm{NiO}$. The components of the mechanical skeleton $\delta$ are joined by three types of grain boundaries $\mathrm{Ni}-\mathrm{Ni}, \mathrm{Ni}-\mathrm{NiO}$ and $\mathrm{NiO}-\mathrm{NiO}$. The components of the magnetic skeleton $\mathcal{S}^{\prime}$ are $\mathrm{Ni}$ nanocrystals joined by $\mathrm{Ni}-\mathrm{Ni}$ grain boundaries. The magnetic labyrinth $\mathcal{L}^{\prime}$ is defined via $\mathcal{L}^{\prime}+\boldsymbol{S}^{\prime}=\boldsymbol{M}^{\prime}$ but is not indicated. The characteristic lengths $d_{\mathrm{NC}}^{\prime}, \overline{d_{\mathrm{NC}}^{\prime}}$, and $d_{\mathrm{CC}}^{\prime}$ are defined with respect to $\mathcal{S}^{\prime}$ and $\mathcal{L}^{\prime}$ in analogy to Fig. 1.

increased. The latter determine the collective effects in the system, similarly as magnetoelastic energy, which has less significance for $\kappa_{S}^{-1}<\kappa_{M}^{-1}$.

Suppose for the sake of simplicity that no Ni-Ni grain boundaries exist, i.e., $\mathcal{S}^{\prime}$ consists of individual $\mathrm{Ni}$ nanocrystals. Because now no Néel or Bloch walls between nanocrystals are needed to minimize crystalline anisotropy of the magnetic skeleton, it could be assumed that each $\mathrm{Ni}$ nanocrystal is magnetized in a magnetically weak direction, similarly as in ferromagnetic micrometer-sized powders or in ferrofluids. ${ }^{35}$ However, the area $A$ spanned by $\partial_{i} \rho^{\prime}$ and being covered by magnetic charge is considerably larger in NS ferromagnets and therefore the characteristic length $\kappa_{S}^{-1}$ is here much more important. Screening of magnetic charge will not only be realized over the small scale $\bar{d}_{\mathrm{NC}}^{\prime}$ (see Fig. 5) but also across Ni nanocrystals.

The point here is that the assumption "magnetically weak directions of adjacent nanocrystals are stochastically distributed" implies that magnetic surface charge at adjacent boundaries is also stochastically distributed and therefore is badly described by dipolar layers. As a consequence, one gets larger-range interactions implying an increase of magnetostatic energy with respect to the aligned case. Suppose now that the magnetization of the central part of a nanocrystal is aligned along a magnetically weak direction. In that case each nanocrystal may be decorated with a superficial Néel wall of a rotation angle $\Theta$ and depth $\Theta \kappa_{S}^{-1}$ in order to obtain a smooth magnetization distribution between adjacent nanocrystals.
This yields for $\theta \kappa_{S}^{-1} \ll d_{\mathrm{NC}}^{\prime}$ effectively the volume ratio,

$$
r=1-6 \Theta \kappa_{S}^{-1} / d_{\mathrm{NC}}^{\prime},
$$

to optimize crystalline anisotropy. Using $\Theta \cong \pi / 6$ one obtains $r \cong \frac{5}{6}$. Observe that the Neel walls contain now the extra magnetostatic energy needed to adjust the magnetization configuration to the weak directions.

This implies that we obtain for the ratio of gain of crystal anisotropy $\left(-\Delta E_{K}\right)$ to loss of magnetostatic $\left(\Delta E_{S}\right)$ energy of such a configuration the rough estimate

$$
\Delta E_{K} / \Delta E_{S} \cong\left(\kappa_{S} / \kappa_{K}\right)^{-2} \frac{r}{1-r} \cong \frac{1}{6},
$$

where the factor $\Theta^{2}$ cancels in the nominator and denominator. This implies $\Delta E_{K}+\Delta E_{S}>0$, i.e., loss of magnetostatic energy dominates, and therefore crystalline anisotropy again will play no role.

For the case that nonometer-sized crystals have an approximate spherical shape the arguments given above imply that the $\mathbf{N}$ field will extend more or less smoothly over the magnetic skeleton and Hopf's invariant may be computed in a similar fashion as indicated in Sec. II, except that now disclinations may have support in magnetic and nonmagnetic material as well. Observe, however, that the presence of nonmagnetic material allows antiparallel order-parameter configurations at interfaces bounded by disclination loops of half integer strength $S$, similarly as in liquid crystals (see Sec. II) because the dominating exchange energy (6) vanishes there. In contrast to integer valued disclination loops the latter are essentially confined to move in nonmagnetic material. The presence of half-integer disclination loops allows the formation of energetically favorable branch points in the disclination network and therefore the situation is different with respect to that, where $\partial_{i} \mathcal{M}=\emptyset$.

It should be recalled that surface coated NS Ni may have some similarity with the magnetic granular solids consisting of nanometer-sized metal granules embedded in an insulating matrix (Liou and Chien, ${ }^{36}$ Gavrin and Chien ${ }^{37}$ ), e.g., $\mathrm{Ni}-\left(\mathrm{SiO}_{2}, \mathrm{Al}_{2} \mathrm{O}_{3}\right)$. Particle sizes range between 1 and $10 \mathrm{~nm}$, and the insulating matrix extends over $\sim 50 \mathrm{vol} . \%$, which is certainly larger than in the case of surface coated Ni. However, the order of magnitudes in quenched ferrofluids consisting, e.g., of magnetite $\left(\mathrm{Fe}_{3} \mathrm{O}_{4}\right)$ particles studied by Luo et al. ${ }^{10}$ are considerably different. Particle sizes are of the order of $\sim 5$ $\mathrm{nm}$ and nonmagnetic coats $\sim 2 \mathrm{~nm}$ thick, whereas packing fractions range from $\eta=0.002-0.04$. Interaction in such systems can be approximated by magnetic dipole interaction and, as Luo et al. ${ }^{10}$ show, clear features of random crystalline anisotropy similar to what is found in amorphous ferromagnets are observed.

An interesting problem with NiO-surface-coated $\mathrm{Ni}$ crystals in NS material is the behavior of antiferromagnetism if it still exists. Because the latter is now confined to the labyrinthine structure $\mathcal{L}^{\prime}$ it may reflect the geometric properties of this object. In case that $\mathrm{NiO}$ is in an amorphous state crystalline anisotropy may also play no role. Within a semiclassical approach antiferromagnetism may then be described, if it still exists, by the Hamiltonian $\mathscr{H}_{J}$ 
given by (6). ${ }^{15}$ In addition, Hopf's invariant may be computed via (15) and (15'), where $\mathcal{M}$ represents the magnetic labyrinth $\mathcal{L}^{\prime}$ and $\mathbf{A}$ and $\mathbf{B}$ are fictitious vector potential and magnetic induction field defined according to Kundu and Rybakov. ${ }^{14}$ However, now $\partial_{i} \mathcal{L}^{\prime}$ has a complex geometric character and is not simply connected, and besides that boundary conditions at $\partial_{i} \mathcal{L}^{\prime}$ are less stringent and therefore a similar analysis as displayed in connection with (20) is not any more reasonable. Furthermore, the time change of $Q_{\mathscr{H}}$ may be computed according to (21), but $\left(21^{\prime}\right)$ is now not well defined.

Antiferromagnetism in NS materials occurs in $\mathrm{FeF}_{2}$, and $\mathrm{CoO}{ }^{1}$ Its theoretical analysis is simplified with respect to ferromagnetism in $\mathrm{Fe}, \mathrm{Co}$, and $\mathrm{Ni}$, because now $\mathscr{H}_{S} \equiv 0$. In addition crystal anisotropy implying the presence of antiphase boundaries in PC material will also play no role in NS antiferromagnets, if $\kappa_{K}^{-1} \gg d_{\mathrm{NC}}$ applies, and antiferromagnetism is not destroyed in $\mathcal{L}^{\prime}$ due to its frustration in a modified structural short-range order. Accordingly such systems may be considered as ideal Heisenberg antiferromagnets. If it is assumed that $\mathcal{L}$ is nonmagnetic one must still make certain assumptions about the boundary conditions at $\partial_{i} \mathcal{\rho}$ and $\partial_{e} M$, in order to compute the defect structure corresponding to (6). This problem has not been analyzed so far.

\section{E. Two-step thermal magnetization disordering}

Eventually we consider briefly the two-step magnetization disordering ${ }^{2,3}$ mentioned in the Introduction. According to the arguments presented at the beginning of this section, the exchange coupling and magnetization (volume) density in the CS material of NS Ni should be reduced with respect to crystalline $\mathrm{Ni}$. On account of that it will disorder below $T_{C}$. However, due to the coupling of magnetic moments at the boundary between the domains $\mathcal{L}$ and $\mathcal{S}$ of the NS material, disordering will not occur in the form of a phase transition. The point is that the magnetic moments within the CS material $(\mathcal{L})$ may be described to form a quasi-two-dimensional Heisenberg magnet subject to external fields (the stray fields and the boundary conditions), which stabilize the order parameter in this subsystem at $T>0$. However, due to the fact that $\mathcal{L}$ represents a multiconnected quasi-2D space (with a Hausdorff dimension $d_{H}>2$ ) it is possible that even without external fields long-range order in $\mathcal{L}$ is possible for $T<T_{\mathrm{CS}}<T_{C}$. In that case it would be plausible to associate the magnetization reduction observed by Kisker ${ }^{2}$ at $\sim 85 \mathrm{~K}$ below $T_{C}$ with a smoothed out phase transition at $T_{\mathrm{CS}} \cong T_{C 1}=T_{C}-85 \mathrm{~K}$. If that holds to be true, a qualitative change of the situation for temperatures $T_{\mathrm{SC}}<T<T_{C}$ arises, because there the CS material may be assumed to be paramagnetic and provide efficient magnetic shielding ${ }^{21}$ of the surface magnetic charge on the nanocrystals of $\mathcal{S}$. In the case that the CS material $(\mathcal{L})$ is highly permeable the magnetism is essentially confined to $\mathcal{f}$ without severe boundary conditions at $\partial_{i} \rho$. This implies that the significance of crystalline anisotropy may be increased depending on the crystallographic structure of $\mathcal{~}$. It should be observed that even a small exchange con- stant $\left(J_{S}\right)$ between adjacent nanocrystals does not imply a considerable reduction of $T_{C}$ of the NS ferromagnet. The point is that even for $T_{\mathrm{CS}} \ll T_{C}$, but $\bar{d}_{\mathrm{NC}} \ll d_{\mathrm{NC}}$, an effective decoupling of adjacent nanocrystals is only possible once the magnetic correlation length $\left(\xi_{\mathrm{CS}}\right)$ of the labyrinthine domain satisfies $\xi_{\mathrm{CS}}<\bar{d}_{\mathrm{NC}}$.

Let us point out that the scenario indicated above is not necessarily realized experimentally due to the observation of grain growth at temperature $T \gtrsim T_{C 1}$ (Refs. 2 and 3) because in the absence of that process $T_{C 1} \cong T_{C}$ may be observed, i.e., a reduction of the Curie temperature in NS Ni. The origin of the latter behavior would be super paramagnetic behavior of nanocrystals, at least of those belonging to the small sized part of the crystal size distribution function.

Consider briefly the fate of the topological theory of magnetism upon approaching the phase transition at $T_{C}$. Obviously there is a competition between $\kappa_{S}^{-1}$ and the magnetic correlation length $\xi$. Due to $M_{S}(T) \sim \tau^{\beta}$ and $\xi \sim \tau^{-v}$, where $\tau \equiv\left|1-T / T_{C}\right|$, and $\beta$ and $v$ are critical exponents with $\beta \leq v, \xi \geq \kappa_{S}^{-1}$ sufficiently close to $T_{C}$ will arise. In that case critical phenomena will be dominated by $\kappa_{S}^{-1}$ and due to the long-range magnetostatic interaction logarithmically modified mean-field behavior i.e., $\beta=v=1 / 2$ may be observed. Such a crossover, however, occurs only relatively close to $T_{C}$. Further below $T_{C}$, where $\xi<\kappa_{S}^{-1}$ holds, critical phenomena will not optimize the magnetostatic energy, and therefore all types of magnetization topologies, which can be excited thermally are possible. The problem is therefore to find out if obstruction to topology changing processes also vanishes because this would imply that hysteresis loops already at temperature below $T_{C}$ collapse to lines, which is unlikely. Presumably, high-energy short-range spin fluctuations reduce topological obstruction continuously upon approaching $T_{C}$, without ever vanishing.

\section{THE COERCIVE FORCE}

In the following part the problem of the coercive force $H_{c}$ in ns material is approached. According to Refs. 2 and 3 one obtains $H_{c}^{0} \sim 100 \mathrm{Oe}$ and $\sim 60$ Oe for NS Ni without and with surface coating, respectively extrapolated to $0 \mathrm{~K}$, whereas the theoretical value due to crystalline anisotropy is $\sim 1600 \mathrm{Oe}$. In polycrystalline $\mathrm{Ni}, H_{c}$ is of the order of a few oersteds. ${ }^{7}$ The coercive force $H_{c}$ for NS Ni without surface coating drops to $\sim 5 \mathrm{Oe}$ at $\sim 50 \mathrm{~K}$ and stays constant at a value of $\sim 10$ Oe far beyond ambient temperature. Surface coated NS Ni shows less pronounced temperature dependence of $H_{c}$, dropping approximately according to an exponential law $H_{c} \sim H_{c}^{0} \exp [-(\ln 2 / 50) T K]$ to $\sim 160$ Oe at $\sim 100 \mathrm{~K}$, and approaching $\sim 70$ Oe at ambient temperature in a linear fashion. ${ }^{2,3}$

\section{A. Intrinsic and extrinsic motion of disclinations}

An interpretation within the preceding theory will now be attempted. First, on account of the absence of Bloch and Néel walls in the system, remagnetization processes 
do not occur over the motion of domain walls, ${ }^{4}$ but via the intrinsic and extrinsic motion of disclinations. Intrinsic and extrinsic motion of disclinations refer to a polarization reversal $\left[S_{i}^{\prime} \rightarrow-S_{i}^{\prime}\right.$ in (53)] in their cores and a spatial displacement of their cores, respectively, and qualitatively correspond to rotational and domain-wall motion, respectively, in classical micromagnetics. ${ }^{6}$ Note that the origin of Brown's paradox ${ }^{38}$ in polycrystalline samples lies in the fact that no nucleation of domain walls is needed, but that those are already present at sharp corners, edges, etc., of the sample, whereto they have been forced to retreat by a strong magnetic field, and thus act as seeds of domain structures. In a reversed applied field they can easily be set in motion and coercive force will mainly depend on the distribution of pinning centers. In small monodomain particles domain walls do not exist and therefore they will approach the theoretical value of $H_{c}$ corresponding to some rotational mode reversal. ${ }^{7}$ Seeds of magnetization structure in the present problem are partly classified by the equivalence classes of the texture group $\pi_{3}\left(S^{2}\right)$ and may consist of entangled disclination loops constrained by structural anomalies of NS material.

Magnetization reversal may occur over intrinsic and extrinsic motion of disclinations, and the respective coercive fields will depend on the size of disclination cores and the strength of topological obstruction for crossing processes in the network of disclinations (see Sec. IV), respectively. A rough estimate of $H_{c}^{(i)}$ for intrinsic motion can be obtained by viewing the core of a disclination as a thin cylinder of radius $r_{c}$. Then a change of polarization, e.g., described by $\left(52^{\prime}\right)$ over $S^{\prime} \rightarrow-S^{\prime}$, via some appropriate rotational motion (see, e.g., Träuble ${ }^{7}$ ) yields

$$
H_{c}^{(i)} / 10^{3} \mathrm{Oe} \cong 3.09 \sqrt{2 \pi} /\left(r_{c} \kappa_{S}\right)^{2} .
$$

Using $r_{c} \cong \pi \kappa_{S}^{-1}$ we obtain $H_{c}^{(i)} \cong 780$ Oe, which can be considered as an upper $T$-independent bound of $H_{c}$ in $\mathrm{Ni}$ in the case where it is modeled as an ideal Heisenberg magnet. For $r_{c}>\pi \kappa_{S}^{-1}, H_{c}^{(i)}$ diminishes, but an increase of $r_{c}$ cannot be easily driven by $H$, because of $\kappa_{S}^{-1} / \kappa_{H_{c}^{(i)}}^{-1} \ll 1$. In addition, one has a bottleneck effect, i.e., $r_{c}$ in (59) is governed by the tightest part of the tube. On account of the experimental observations $s^{2,3}$ and the estimate of $H_{c}^{(i)}$ given we expect that $H_{c}^{(e)} \leq H_{c}^{(i)}$. The point now is that $H_{c}^{(e)} \rightarrow 0$ applies for an ideal Heisenberg magnet, where no pinning exists and topological obstruction is absent $(\eta=\infty$, see Sec. III). For the case $\eta=0$, the topological character $(\alpha)$ of a magnetization configuration is fixed once for ever and can be represented by the pair $\left(H_{c}^{(\alpha)}, M_{R}^{(\alpha)}\right)$, with $H_{c}^{(\alpha)} \geq 0, M_{R}^{(\alpha)} \leq M_{S}$. For a NS ferromagnet we expect that $H_{c}^{(e)}$ determines $H_{c}$.

\section{B. Surface-coated NS Ni versus NS Ni}

From the observation that surface-coated NS Ni has an increased coercive force with respect to NS Ni may either follow that the topological entanglement of disclinations is stronger there, or that crossing processes of disclinations are faced with severer obstructions, or that disclinations are less mobile due to pinning. Presumably all three mechanisms apply because a reduction of exchange energy implies a more nonuniform magnetic state leading to more effective pinning and to the possibility of more complex entanglements. The situation is slightly counterintuitive because releasing constraints in the system (decoupling exchange between the joints of the mechanical skeleton $\mathcal{S}$, i.e., replacing $\mathcal{S}$ by $\mathcal{S}^{\prime}$ ) leads to stronger obstruction of magnetization processes, i.e., increased $H_{c}$. As a matter of fact, this may be viewed as an example of Braess' paradox ${ }^{39}$ applying to noncooperative games and to systems where Kirchhoff's law (e.g., $\nabla \cdot B=0$ ) holds. ${ }^{40}$ Pinning effects may be associated with internal surface structures in the form of pores. Note in this respect the possibility of existence of half-integer strength disclination loops confined to nonmagnetic material acting as natural pinning centers when incorporated in a branched disclination network as indicated in Sec. V. In addition, in the case of $d_{\mathrm{CC}}>d_{\mathrm{NC}}$ the crystallographic weak directions in single crystals may be replaced by a bundle of "weak" curves (whose structure is determined by $\mathfrak{f}^{\prime}$ ) along which crystalline anisotropy energy is optimized. This may lead to a trapping of magnetic flux along a system of entangled curves. A similar concept of weak lines can be developed for the magneto-static energy, for nonspherical nanocrystals and specific structures of $\delta^{\prime}$.

A model distribution [for the $\mathrm{O}(3)$-order parameter in the bulk, $\mathbf{N}(\mathbf{r})$ ] derived from Ref. 14 may be of the form

$$
\begin{aligned}
& \gamma(\mathbf{r})=\sum_{n=1}^{N} S_{n} \arctan \frac{Y-y_{n}}{X-x_{n}}+v(\mathbf{r}), \quad S_{n} \in \mathbb{Z} \\
& \beta(\mathbf{r})=\arccos w(\mathbf{r}),
\end{aligned}
$$

where $R=(X(r), Y(r))$ is defined in (52) and the functions $w(\mathbf{r})$ and $v(\mathbf{r})$ are subject to the boundary conditions

$$
\begin{aligned}
& w\left(\mathbf{r}_{n}\right)=S_{n}^{\prime}, \quad \mathbf{r}_{n} \in \partial C_{n}, \quad n=1, \ldots, M, \\
& w\left(\mathbf{r}_{m}\right)=S_{m}^{\prime}, \quad \mathbf{r}_{m} \in \partial C_{m}, \quad m=M+1, \ldots, N, \\
& w(\mathbf{r})=0, \quad r \in \partial_{e} \mathcal{M}-\left\{\mathbf{r}_{i}\right\}, \\
& \left\{\mathbf{r}_{i}\right\}=\partial_{e} M \cap\left\{\partial C_{n}\right\}_{n=1, \ldots, M}, \\
& \Delta v\left(\overline{\mathbf{r}}_{k}\right)=2 \pi S_{k}, \quad \overline{\mathbf{r}}_{k} \in C_{k}, \quad k=1, \ldots, N .
\end{aligned}
$$

Here $\left\{\partial C_{n}\right\}$ and $\left\{\partial C_{m}\right\}$ denote open-ended and closed disclinations cores in $\mathcal{M}$, respectively, and $C_{m}$ is the cut surface spanned by $\partial C_{m}$. The function $w(\mathbf{r})$ is unique and differentiable assuming extremal values along disclination cores, i.e., $S_{n}^{\prime}, S_{m}^{\prime} \in\{ \pm 1\}$, where $v(\mathbf{r})$ is discontinuous over the cut surfaces as indicated by $(61 \mathrm{~d})$. For $\left(16^{\prime}\right)$ one obtains with $(60)$

$$
Q_{\mathscr{H}} \sim \sum_{n<m} S_{n} S_{m} \frac{1}{2}\left(1-S_{n}^{\prime} S_{m}^{\prime}\right) \Phi\left(\partial C_{n}, \partial C_{m}\right),
$$

where the factor $\frac{1}{2}\left(1-S_{n}^{\prime} S_{m}^{\prime}\right)$ may also be included in the definition of $\Phi$.

This situation is sketched in Fig. 6 for a cross section along the $x y$ plane in the bulk, where disclinations are assumed pairwise linked. Application of a magnetic field 

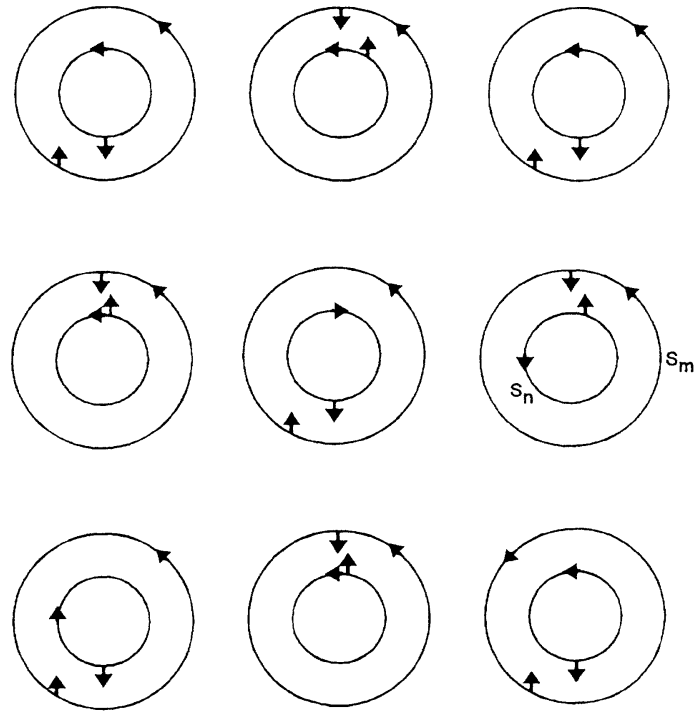

FIG. 6. Schematic representation of a section of a latticelike structure of pairwise entangled disclination loops via a planar cross section in the bulk. Small circles indicate open-ended disclinations and large circles closed loops of disclinations. A round arrow indicates disclination strength $S_{n}$ and $S_{m}$, whereas up and down arrows correspond to $S_{n}^{\prime}, S_{m}^{\prime}=1$, and -1 , respectively.

$H>0$ will yield growth and contraction of large circles surrounding small circles with an up and a down arrow, respectively. Nontrivial topological processes imply change of linking. The detailed form of $\left\{\partial C_{n}\right\}$ and $\left\{\partial C_{m}\right\}$ lines will depend on the structure of the NS ferromagnet and the processing of its magnetic structure. Suppose, for instance, that in surface-coated NS Ni the system of lines $\left\{\partial C_{n}\right\}$ and $\left\{\partial C_{m}\right\}$ is strongly trapped in the labyrinthine domain $\mathcal{L}$ indicated in Fig. 5. Then a change of magnetization upon application of a magnetic field will essentially take place over rotation of $\mathbf{N}(\mathbf{r})$ counter acted by local stray fields. For NS Ni such a trapping of disclinations is less likely because exchange coupling between $\mathcal{f}$ and $\mathcal{L}$ will prevent it; change of magnetization in NS Ni is then a consequence of the sweeping motion of the $\left\{\partial C_{m}\right\}$ line system, which imposes less resistance to an applied field than a constrained rotational motion. This explains qualitatively the increased coercive force of the surface-coated NS Ni with respect to NS $\mathrm{Ni}$.

The theoretical concepts developed so far may not be sufficient to develop a microscopic theory of the $(M, H)$ hysteresis loops of NS ferromagnets because Fig. 6 does not represent the generic case. The latter may consist of an entangled network of disclinations (including branch points) similar to that in liquid crystals or in polymer melts, and some of the theoretical concepts developed for the latter systems may be applied, ${ }^{41}$ e.g., random walks of disclinations, line tension, etc., although such systems do not show the pronounced hysteretic effects of ferromagnets. Accordingly additional ideas are needed to resolve the complexity of the $(M, H)$-hysteresis loops in NS fer- romagnets, and the approach indicated in Sec. V, based on the ideas of Moffatt ${ }^{34}$ may be the right point of departure. Time-dependent phenomena may be studied within the framework of self-organized criticality ${ }^{42}$ as suggested by Sethna et $a l .{ }^{43}$ for the random-field Ising model.

\section{The temperature dependence of $H_{c}$}

Another interesting problem is the temperature dependence of the coercive force. ${ }^{2,3}$ In the authors opinion the strong $T$ dependence of $H_{c}$ at low temperatures, $T<50 \mathrm{~K}$ for NS Ni, and $T \lesssim 100 \mathrm{~K}$ for surface-coated NS Ni, cannot be explained by an intrinsic $T$ dependence of $J, K$, and $M_{S}$, except if that is correlated with a reversible change of the NS material, which is unlikely. Because $H_{c}$ for NS Ni above $50 \mathrm{~K}$ does hardly depend on temperature and the same applies to surface coated NS Ni above $100 \mathrm{~K}$ to a lesser degree, thermal activation barriers can only play a role at low temperatures. A tentative interpretation of the low-temperature behavior of $H_{c}$ is based on the theories developed in Secs. III and IV. In particular it is suggestive that the temperature $T_{m}$ defined in Sec. IV, which marks thermal population of longwavelength dipolar modes in a NS ferromagnet, can be associated with the change over of the $T$ dependence of $H_{c}$ in NS and surface coated NS Ni, observed at $50 \mathrm{~K}$ and $100 \mathrm{~K}$, respectively. An interpretation of $H_{c}$ in the different temperature regimes is based on (36), i.e., $H_{c}$ is correlated to the $T$ dependence of the local-spin resistivity. Suppose, e.g., that $\eta_{t}$ at low temperatures increases with a power law in the form $\eta \sim a+b T^{\alpha_{i}}$ then (36a) and (36b) yield a $1 / T$ and exponential decrease, respectively, of $H_{c}$ with increasing temperature. Accordingly (36) allows a rough fit of the $H_{c}$-versus- $T$ curve observed for surface coated NS Ni. The change over from (36b) to (36a), however, cannot be explained simply by the $T$ dependence of $\eta_{t}$ but must be due to more fundamental properties of disclination entanglements and pinning, and which may be related to the screening properties of dipolar excitations as indicated in Sec. IV and above. In this picture the dipolar modes drive an intrinsic transition in the entanglement structure of the disclination network. For $\mathrm{NS} \mathrm{Ni}$, (36b) may describe the low- $T$ behavior, whereas for $T \gtrsim 50 \mathrm{~K}, H_{c}$ stays roughly constant and therefore (36a) may apply with $\eta_{t}$ roughly constant.

\section{Qualitative estimate of $\eta_{t}$}

Eventually we will make a few remarks with respect to a qualitative evaluation of the $T$ dependence of $\eta_{t}$. In the simplest approach, one may compute $\eta_{t}$ over the Drude formula for the low-frequency conductivity in metals ${ }^{21}$

$$
\sigma_{D}=\sigma_{0}(T) /(1-i \omega \tau)
$$

where $\tau$ is a relaxation time and $\sigma_{0}(T)$ is given by the Einstein relation

$$
\sigma_{0}(T)=e^{2}(\partial n / \partial \mu) D
$$

For $T=0, \partial n / \partial \mu$ yields the density of states $N\left(\epsilon_{F}\right)$ at the Fermi surface and $D=(1 / 3) v_{F} l_{t}$ is the diffusion constant, 
where $v_{F}$ and $l_{t}$ are Fermi velocity and transport free path, respectively. In strongly disordered (glassy) metals the low- $T$ conductivity is dominated by elastic scattering $^{44}$ and the associated quantum interference effects (weak localization, see Ref. 45), not described by $\sigma_{0}(T)$. Due to loss of time-inversion symmetry in a ferromagnet weak localization need not be considered in the following. In NS material $l_{t}$ will scale with $d_{\mathrm{NC}}$, i.e., $l_{t} \gtrsim d_{\mathrm{NC}}$ assuming that nanocrystals have a low density of defects. The precise law will depend on the scattering properties of $\mathcal{L}$, i.e., if grain boundaries impose small- $\left(l_{t}>d_{\mathrm{NC}}\right)$ or large$\left(l_{t} \cong d_{\mathrm{NC}}\right)$ angle electron scattering. For $l_{t} \cong d_{\mathrm{NC}}, \sigma_{0}(T)$ will be dominated by elastic scattering, and only a very weak $T$ dependence of $\sigma$, being due to inelastic scattering (electron-magnon-phonon processes), will develop up to ambient temperature and beyond. The precise law for NS Ni is not known to us but we expect $\sigma(300 \mathrm{~K}) / \sigma(4.2$ $\mathrm{K}) \lesssim 0.5$, from data applying to strongly disordered systems. ${ }^{44}$ The dependence of $\tau$ in (63) on disorder is not known (the standard $\sigma$ model does not reproduce Drude behavior ${ }^{45}$ ) but we expect that $\tau \gtrsim 1_{t} / v_{F}$ applies, i.e., $\tau \gtrsim 10^{-14} \mathrm{sec}$ for NS Ni. In that case we may ignore the imaginary part of $\sigma_{D}$ and set $\eta_{t} \cong 1 / \sigma_{0}(T)$ being only weakly $T$ dependent.

In a more precise theory the $q$ dependence of $\sigma$ should also be taken into account. Using the dielectric constant $\epsilon(\mathbf{q}, \omega)$ defined by

$$
\varepsilon(\mathbf{q}, \omega)=1+\frac{4 \pi e^{2}}{q^{2}} \pi(\mathbf{q}, \omega),
$$

where the irreducible polarization is given by ${ }^{45}$

$$
\pi(\mathbf{q}, \omega)=\frac{\partial n}{\partial \mu} \frac{D(\mathbf{q}, \omega) q^{2}}{D(\mathbf{q}, \omega) q^{2}-i \omega}
$$

one obtains ${ }^{21} \quad \sigma(\mathbf{q}, \omega) \cong-i(\omega / 4 \pi)[\epsilon(\mathbf{q}, \omega)-1]$. For $\operatorname{Im} D(\mathbf{q}, \omega) \cong 0$ this implies

$$
\sigma(\mathbf{q}, \omega)=\frac{\omega^{2} e^{2}(\partial n / \partial \mu) D(\mathbf{q}, \omega)}{D^{2}(\mathbf{q}, \omega) q^{4}+\omega^{2}} .
$$

In the classical regime $D(\mathbf{q}, \omega)$ may be represented in a Taylor series of the form ${ }^{46}$

$$
D(\mathbf{q}, \omega)=D+\sum_{n, m} \gamma_{n m}(\omega \tau)^{n}\left(q l_{\mathrm{tr}}\right)^{m} .
$$

Setting $\eta_{t} \sim \eta\left(q_{c}, \omega_{c}\right)=1 / \sigma\left(q_{c}, \omega_{c}\right)$ as indicated in Sec. III we obtain $\omega_{c} \tau=2 \pi\left(\tau / \tau_{t}\right)$ and $q_{c} l_{\mathrm{tr}} \cong 2 d_{\mathrm{NC}} \kappa_{S}$, where $\tau_{t}$ is defined by (32). Using low-temperature values, i.e., $\tau_{t} \gtrsim h / k_{B} T_{C} \cong 0.6 \times 10^{-13}$ sec for $\mathrm{NS} \mathrm{Ni}$, we get $\omega_{c} \tau \cong \mathrm{O}(1)$ and $q_{c} l_{t} \gg 1$, and for $T \rightarrow T_{C}$ we obtain $\omega_{c} \tau \ll<1$ and $q_{c} l_{t} \ll<1$. Accordingly a cross over phenomena in time at low temperatures and in space at temperatures approaching $T_{C}$ may exist.

In the high-temperature domain we obtain from (65)

$$
\eta_{t} \sim \frac{1}{\sigma_{0}(T)}\left[1+\left(\frac{2}{3 \pi}\left(\tau_{t} / \tau\right)\left(d_{\mathrm{NC}} \kappa_{S}\right)^{2}\right)^{2}\right] .
$$

In the mean-field regime we may set $x_{t}=1$ in (32), and in $\kappa_{S}, \beta=1 / 2$ and obtain a finite $\eta_{t}$ for $T \rightarrow T_{C}$. This result implies very weak $T$ dependence of $\eta_{t}$ over a wide tem- perature regime upon approaching $T_{C}$. In the critical domain we have $\beta \cong \frac{1}{3}, z>2 v \cong 4 / 3$ (Ref. 25) implying $\eta_{t} \sim 1 /\left(1-T / T_{C}\right)^{4 / 3}$. Inserted into (36a) and setting $\alpha_{1}=1$, this yields the power law according to which $H_{c}$ vanishes (within our theory) upon approaching $T_{C}$. Because very close to $T_{C}$, long-range interaction will dominate critical phenomena leading to logarithmically modified mean-field behavior, as has been pointed out at the end of Sec. IV, we expect there $\eta_{t} \sim \ln 1 /\left(1-T / T_{C}\right)$. Note that the present result qualitatively agrees with observations on $\mathrm{NS} \mathrm{Ni,} \mathrm{that} H_{c}$ stays roughly constant for $T \gtrsim 50 \mathrm{~K} .2,3$

The crossover phenomena at low $T$ occuring in time are more difficult to assess. It is known, however, that in some strongly disordered systems similarly, as in insulators, $\sigma(\mathbf{q}, \omega)$ grows with increasing $\omega .{ }^{45}$ According to Mott and Davis, ${ }^{47}$ strong scattering transfers spectral weight from low frequencies to higher frequencies and thus is predicted to lead to non-Drude-like conductivity, e.g., $\sigma \sim \omega$, as observed recently in icosahedral quasicrystals $\mathrm{Al}_{63.5} \mathrm{Cu}_{24.5} \mathrm{Fe}_{12}{ }^{48}$ Because $\omega_{c} \tau$ diminishes with increasing $T$, it implies that $\eta_{t} \sim 1 / \sigma\left(q_{c}, \omega_{c}\right)$ will grow with $T$. Assuming tentatively that $\tau_{t}$ is affected by dipolar excitations as indicated in Sec. IV, one may conclude that for $T>T_{m}$ we have $\omega_{c} \tau \ll<1$, and $T$ independence. $\mathrm{Ob}$ serve that this also requires a modification of the Ansatz (32), which exhibits a much slower change of $\omega_{c}$ with temperature as required here. On account of that it is more reasonable to explain the change over of $H_{c}$ at $T_{m}$ by collective effects driven by dipolar excitations.

For surface-coated NS Ni, the physical situation is more complex because too little is known about the electronic properties of this system. The simplest assumption is that it is a dc insulator on a scale $l>d_{\mathrm{NC}}$, and a conductor for $l<d_{\mathrm{NC}}$. In that case (47) approximately applies for $q<2 \pi / d_{\mathrm{NC}}$. Due to $q_{c}<2 \pi / d_{\mathrm{NC}}$ at low temperatures the qualitative estimates presented for $\mathrm{NS} \mathrm{Ni}$ above should also apply, with the parameters $l_{t}$ and $\tau$ redefined in an appropriate fashion. A considerable modification of the $T$ dependence of $H_{c}$ at temperatures, where $q_{c}>2 \pi / d_{\mathrm{NC}}$ applies is expected, implying that $q_{c} \cong 2 \pi / d_{\mathrm{NC}}$ tentatively may be associated with $T_{m} \cong 100$ $\mathbf{K}$.

\section{CONCLUDING REMARKS}

It is pointed out that our interpretation of the $T$ dependence of $H_{c}$ is certainly not the only one. In particular Kisker $^{2}$ and Schaefer et al. ${ }^{3}$ apply to $H_{c}$ a statistical theory of Néel, Stoner, and Wohlfahrt (NSW) (see, e.g., Wohlfahrt ${ }^{49}$ ) using a competition between crystalline and form anisotropy. Naturally, such effects will play some role because particle sizes are not sufficiently small, so that not only collective effects play a role, as suggested in the present paper. For instance in NS Co, collective effects are less important due to $\kappa_{S}^{-1} / \kappa_{K}^{-1} \sim \frac{1}{2},{ }^{6}$ being much larger than in NS Ni and Fe. Presumably, however, collective effects are strong enough to invalidate an independent particle model. Let us point out that the NSW model does not account for particle interaction and 
therefore does not describe nonsymmetric minor hysteresis loops. For further deficiencies of this model and how they may be circumvented see Mayergoyz. ${ }^{26} \mathrm{Ob}$ serve that the local structure of NS material is not well understood, e.g., it is not known what the characteristic shape of nanocrystals after compaction and sintering is. The manifold $\mathcal{M}$ and its boundary $\partial \mathcal{M}$ representing the NS specimen are certainly multiconnected spaces. A representation of $\mathcal{M}$ as a skeleton $(\mathscr{S})$ and labyrinthine structure $(\mathcal{L})$ as indicated in Fig. 1 is a paradigm. Due to the fact that $\partial \mathcal{M}$ and $\partial_{i} \mathcal{S}$ are not simply connected twospaces, magnetic flux may be trapped inside $M$ in such a fashion that even strong applied fields are unable to pull it out of the system. A trapped flux of this kind would play then a similar role as reversed domains at edges and corners in polycrystalline samples, imply reduced coercive forces, and invalidate an independent-particle model.

Our theoretical interpretation of the coercive force in NS ferromagnets is based on the concept of intrinsic and extrinsic motion of disclinations yielding $H_{c}^{(i)}$ and $H_{c}^{(e)}$, respectively, as indicated in Sec. VI. $H_{c}^{(i)}$ is closely related to the micromagnetic description of magnetization reversal in small particles. Here it is referred to the polarization reversal in the core of a disclination. It can be computed via a classical stability analysis and is slightly reduced by tunneling processes. In contrast $H_{c}^{(e)}$ refers to the spatial motion of the disclination cores in a network of disclinations. It compares to the motion of domain boundaries and is affected by pinning and topological obstruction. We propose that pinning in NS Ni at $T<50 \mathrm{~K}$ is successively released by thermal excitation of dipolar modes, and that $H_{c}$ for $T \gtrsim 50$ is essentially determined by topological obstruction as described by $H_{c}^{(e)}$. The theory developed for $\boldsymbol{H}_{c}^{(e)}$ differs essentially from that applying to $H_{c}^{(i)}$ because it cannot be based on a classical stability analysis within a continuum or, say, a micromagnetic approach. Because crossing processes of disclinations are accompanied by the temporal formation of singularities, they can only be described by additional parameters derived from the microscopic laws of magnetism. Within a theory of the quasistatic change of topology the characteristic time parameter seems to be $\eta_{t} \sim \eta\left(q_{c}, \omega_{c}\right)$ (non-local-spin resistivity) with $\omega_{c}^{-1}$ the characteristic time scale to accomplish a topology changing process over the core size $\sim q_{c}^{-1}$ of a disclination. The main conclusion is therefore that the characteristic lengths (1) to (3) have to be supplemented by a characteristic time scale, which physically is expressed in $\eta_{t}$ governing via $\left(21^{\prime \prime}\right)$ topology changing processes in the quasistatic approximation.

In this context we would like to point out that a measurement of the time dependence of the relaxation of remanent magnetization in NS ferromagnets may be useful and will allow comparison with the observations on ferrofluids ${ }^{10}$ and ideal Heisenberg magnets. ${ }^{50}$ In particular, the authors of the latter paper have developed an interesting theory of relaxation processes, based on percolation theory and mesoscopic domains, which applies rather successfully to EuS. Their basic formula for the time dependence of remanent magnetization is

$$
M_{R}(t) \sim \sum_{s=0}^{\infty}\left[s n_{s}\right] \exp \left(-t w_{s}\right),
$$

where $n_{s}$ is the domain size $(s)$ distribution and $w_{s} \sim \exp \left(-\delta E_{s} / k_{B} T\right)$ the corresponding relaxation rate for activated behavior. In contrast to models of domain rotation, where, e.g., superparamagnetic relaxation $\left(\delta E_{s} \sim s\right)$ is assumed, they take $\delta E_{s} \sim \Delta / s$, which implies relaxation processes via geometrically confined magnons. Because relaxation processes in NS ferromagnets in our opinion should involve the motion of disclinations and topological reconstruction of the order parameter, the formula given above does not necessarily apply. Although relaxation processes are ultimately driven by magnons, the picture of domains and their percolative distribution may have to be replaced by a more suitable scenario. For instance one may take for $n_{s}$ the distribution of knots and links confined to effective domains of size $s$ representing the nonequilibrium configuration. Relaxation processes are then supposed to take place within individual knots and links releasing a net magnetization proportional to $s$. A computation of $M_{R}(t)$, however, still requires assumptions as to the form of $n_{s}$ and $\delta E_{s}$ as a function of $s$.

The basic qualitative assumptions about the structure of NS material have been schematically illustrated in Figs. 1 and 5 for NS Ni and surface-coated NS Ni, respectively. In order to obtain a more reliable picture of the structure of NS materials from a theoretical point of view a model of its formation should be developed. On the other hand one may also study their electrical properties experimentally, in particular, at low temperatures. Specifically for surface-coated NS Ni this may give some indication about the structure of $\mathcal{S}$ and $\mathcal{S}^{\prime}$ indicated in Fig. 5. For instance, the magnetic skeleton $\mathcal{S}^{\prime}$ may also be identified with the "metallic" skeleton $\mathcal{S}^{\prime \prime}$, defined in an analogous fashion based on collective electronic properties. Metallic conductivity of the sample may then be an indication of the presence of an infinitely extended cluster in $\mathcal{f}^{\prime \prime}$, supposing that the CS material in Fig. 5 is nonconducting. Note, however, that the CS material is certainly not stoichiometric NiO but a low-density highly defective compound with semiconducting properties.

It is pointed out that NS metals are strongly disordered systems, where low-temperature electronic and thermal transport properties are governed by elastic relaxation times and therefore quantum interference effects may play an important role. ${ }^{44,45}$ This applies also to magnon and phonon excitations, whose low-temperature damping is mainly governed by elastic relaxation and may affect the temperature laws of the saturation magnetization, specific heat, and thermal transport. The point is that a significant change of the quasiparticle nature automatically will change the respective macroscopic temperature laws. From this point of view it is interesting that the low-temperature saturation magnetization in NS Ni and PC Ni obey the same $T^{2}$ law. Because this law would require an effective low- $q$ magnon dispersion $\omega \sim q^{3 / 2}$, it cannot be derived from a simple Heisenberg model, but must be a consequence of the original Hubbard model used to describe itinerant magnetism in transition metals. 
Due to the modified dispersion elastic relaxation $\left[\tau_{e}(q)\right]$ of magnons must display a different $q$ dependence as in localized magnetism. Accordingly there exists the possibility that the relative macroscopic temperature laws in NS and PC ferromagnets differ for itinerant and localized magnetism. This does affect the micromagnetic theory of NS ferromagnetism developed in this paper in a qualitative fashion because the gradient operator $\nabla$ in (6) has to be replaced by the operator $\nabla^{\prime}$, which may be defined by its operation on the complete set of functions $\left\{e^{i \mathbf{k} \cdot r}\right\}$ in the form $\nabla^{\prime} e^{i \mathbf{k} \cdot \mathbf{r}}=a^{-1 / 4}(i k)^{3 / 4}(\mathbf{k} / k) e^{i \mathbf{k} \cdot \mathbf{r}}$. This implies that exchange interactions are effectively reduced and may be expressed via the corresponding characteristic length $\kappa_{S}^{\prime-1}=\left(2 \pi a \kappa_{S}\right)^{1 / 9} \kappa_{S}^{-1}$. Due to $\left(2 \pi a \kappa_{S}\right)^{1 / 9} \cong 1$ the effect is apparently small, but it will also change the interaction law of topological defects. Because we have essentially only used the topological properties of the theory together with some characteristic length units, our conclusions are only little affected by this qualitative change.

Further information about the structure of NS ferromagnets may be obtained via their behavior under plastic deformation. Although this does not produce work hardening, ${ }^{1}$ it may have a certain effect on their microscopic structure (implying, e.g., a change of magnetic and electrical properties) and thus allow to study the reliability of the models sketched in Figs. 1 and 5. For instance, does plastic deformation affect the shape and connectedness of individual nanocrystals and grain boundaries, or alternatively does it affect only the CS material and the relative arrangement of nanocrystals? In the first case, Figs 1 and 5 may apply with $\delta^{\prime} \sim \mathcal{S}$ in Fig. 5, whereas in the second case large and small angle (Ni-Ni) grain boundaries will not exist, and this should have an effect on the macroscopic electronic properties of the samples (metallic conduction in the first case, insulating behavior in the second case). Observe that the mere fact of absence of work hardening in NS materials, ${ }^{1}$ may imply selfsimilarity of their microscopic structure under plastic deformation and therefore also invariance of their magnetic and electrical properties. This conjecture seems to be plausible in view of the fact that during the process of formation of NS material via compaction the aggregate of nanocrystals has already been subject to a hierarchy of stress fields. If this hypothesis holds true, the construction of models of NS materials could be facilitated and their mode of plastic deformation could be specified more easily.

The main problem of micromagnetics in NS ferromagnets from a mathematical point of view is the development of an efficient calculus to handle the intrinsic nonlinearities of the formalism in the absence of domain walls. In the presence of Bloch walls there exist almost no magnetic charging effects in the bulk, whereas in a NS ferromagnet this cannot be avoided, and field configurations are, in general, magnetically charged.
Part of the problem has been circumvented in this paper by the introduction of topological defects with the properties of disclinations, and the hypothesis that magnetic configurations essentially can be described by their core geometry. In this approach it is postulated that the core has a linear dimension of $\sim \pi \kappa_{S}^{-1}$ and that it is magnetically charged, whereas the field in between disclination cores is essentially unchanged. Accordingly the conjecture should be proven that the energetically favorable magnetization configurations are such that a segregation of $\{\mathbf{M}\}$ into $\{\boldsymbol{\nabla} \cdot \boldsymbol{M} \neq 0\}$ and $\{\boldsymbol{\nabla} \times \mathbf{M} \neq 0\}$ takes place forming textures that can be described by disclinations with $\boldsymbol{\nabla} \cdot \mathbf{M} \neq 0$ concentrated in their cores and $\boldsymbol{\nabla} \times \mathbf{M} \neq 0$ in the space in between.

In conclusion, we have shown that magnetism in NS material has some interesting theoretical aspects and may be brought in connection with the theory of topological defects. In particular, it is postulated that polelike singularities of the magnetization should be visible at the surface appearing essentially in pairs, and if this is not the case pores should play a significant role. The coercive force may be interpreted as a measure of the strength of topological entanglement, and pinning by immobile half integer strength disclinations in NS Ni and in surfacecoated NS Ni, respectively. Remagnetization processes are not driven by Bloch or Néel wall motion, as is usual, but by disclinations and require ultimately disentanglement of topological defects. The latter represent the texture of magnetization associated to the semiclassical order parameter $\mathbf{M}$ and Hopf's invariant associated to $\pi_{3}\left(S^{2}\right)$. The $T$ dependence of $H_{c}$ below $50 \mathrm{~K}$ and $100 \mathrm{~K}$ for NS Ni and surface-coated NS Ni, respectively, has been tentatively interpreted in terms of the $T$ dependence of the local (spin) conductivity, whereas the high- $T$ domain of $H_{c}$ may reflect an intrinsic transition of the entanglement structure of disclinations. It is suggested that collective magnetization processes are dominant, i.e., statistical averaging over crystalline anisotropy and magnetic form anisotropy must be subject to strong correlation over the sample in order to provide a screening of magnetic stray fields. This implies that the Neel-StonerWohlfahrt ${ }^{49}$ theory of magnetism, even when applied to micrometer-sized particle aggregates, needs a nontrivial extension if applied to NS ferromagnets, which cannot be simply a scale transformation.

\section{ACKNOWLEDGMENTS}

The authors acknowledge fruitful discussions with $\mathbf{R}$. Birringer, H. Gleiter, H.-E. Schaefer, and R. von Rüden for designing the figures. One of the author's (A.H.) stay at the Instituto de Fisica of Universidade Federal do Rio Grande do Sul was arranged within the agreement DAAD (Germany)-CNPq (Brazil). This work was supported by Deutsche Forschungsgemeinschaft under Grant No. Ho 841/7-1 and CNPq (Brazil). 
${ }^{1}$ H. Gleiter, Prog. Mater. Sciences 33, 223 (1989).

${ }^{2}$ H. Kisker, diploma thesis, University of Stuttgart, 1991 (unpublished).

${ }^{3}$ H.-E. Schaefer, H. Kisker, H. Kronmüller, and L. Würschum, Nanostr. Mater. 1, 523 (1992).

${ }^{4}$ R. S. Tebble, Magnetic Domains, Methuen's Monographs on Physical Subjects (Methuen, London, 1969).

${ }^{5}$ E. P. Wohlfahrt, in Ferromagnetic Materials Vol. 1, edited by E. P. Wohlfahrt (North-Holland, Amsterdam, 1980), p. 3.

${ }^{6} \mathrm{H}$. Kronmüller, in Moderne Probleme der Metallphysik II, edited by Alfred Seeger (Springer, Berlin, 1966), p. 24.

${ }^{7}$ H. Träuble, in Moderne Probleme der Metallphysik II (Ref. 6), p. 157.

${ }^{8}$ F. E. Luborsky, in Ferromagnetic Materials Vol. 1 (Ref. 5), p. 453.

${ }^{9}$ R. Schäfer, A. Hubert, and G. Herzer, J. Appl. Phys. 69, 5325 (1991).

${ }^{10}$ W. Luo, S. R. Nagel, T. F. Rosenbaum, and R. Rosensweig, Phys. Rev. Lett. 67, 2721 (1991).

${ }^{11}$ N. D. Mermin, Rev. Mod. Phys. 51, 591 (1979).

${ }^{12} \mathrm{R}$. Bott and L. W. Tu, Differential Forms in Algebraic Topology, Graduate Texts in Mathematics Vol. 82 (Springer, New York, 1982).

${ }^{13}$ H. J. de Vega, Phys. Rev. D 18, 2945 (1978).

${ }^{14}$ A. Kundu and Yu. P. Rybakov, J. Phys. A 15, 269 (1982).

${ }^{15}$ A. Holz, Physica A 182, 240 (1992).

${ }^{16}$ Y. Bouligand, J. Phys. (Paris) 35, 959 (1974).

${ }^{17}$ Y. Bouligand, B. Derrida, V. Poénaru, Y. Pomeau, and G. Toulouse, J. Phys. (Paris) 39, 863 (1978).

${ }^{18}$ M. A. Berger and G. B. Field, J. Fluid Mech. 147, 133 (1984).

${ }^{19}$ A. Hubert, J. Phys. (Paris) Colloq. 49, C8-1859 (1988).

${ }^{20}$ L. H. Kauffman, On Knots (Princeton University Press, Princeton, 1987).

${ }^{21}$ J. D. Jackson, Classical Electrodynamics (Wiley, New York, 1975).

${ }^{22}$ W. J. Zakrzewski, Low Dimensional Sigma Models (Adam Hilger, Bristol, 1989).

${ }^{23}$ Ian Affleck, Phys. Rev. Lett. 46, 388 (1981).

${ }^{24}$ P. Arnold and L. McLerran, Phys. Rev. D 37, 1020 (1988).

25 J. Zinn-Justin, Quantum Field Theory and Critical Phenomena (Clarendon, Oxford, 1989).

${ }^{26}$ I. D. Mayergoyz, Mathematical Models of Hysteresis (Springer, New York, 1991).
${ }^{27}$ R. M. Bozorth, Ferromagnetism (Van Nostrand, New York, 1951).

${ }^{28}$ J. M. Luttinger and L. Tisza, Phys. Rev. 70, 954 (1946).

${ }^{29}$ M. H. Cohen and F. Keffer, Phys. Rev. 99, 1135 (1955).

${ }^{30}$ R. M. White, Quantum Theory of Magnetism (McGraw-Hill, New York, 1970).

${ }^{31} \mathrm{P}$. Wachter, in Handbook on the Physics and Chemistry of Rare Earths, edited by K. A. Gschneider and L. Eyring, (North-Holland, New York, 1979), p. 507.

${ }^{32}$ F. Liu, M. R. Press, S. N. Khanna, and P. Jena, Phys. Rev. B 39, 6914 (1989).

${ }^{33}$ H. Haken, Synergetics, An Introduction (Springer, Berlin, 1983).

${ }^{34}$ H. K. Moffatt, Nature (London) 347, 367 (1990).

${ }^{35}$ R. Rosensweig, Ferrohydrodynamics (Cambridge University, Cambridge, England, 1985).

${ }^{36}$ S. H. Liou and C. L. Chien, J. Appl. Phys. 63, 4240 (1988).

${ }^{37}$ A. Gavrin and C. L. Chien, J. Appl. Phys. 67, 938 (1990).

${ }^{38}$ A. Holz, Z. Angew. Phys. 23, 170 (1967); J. Appl. Phys. 41, 1095 (1970).

${ }^{39}$ D. Braess, Unternehmensforsch. 12, 256 (1968).

${ }^{40} \mathrm{~J}$. E. Cohen and P. Horowitz, Nature (London) 352, 699 (1991).

${ }^{41}$ M. Doi and S. F. Edwards, The Theory of Polymer Dynamics (Clarendon, Oxford, 1986).

${ }^{42}$ P. Bak, C. Tang, and K. Wiesenfeld, Phys. Rev. Lett. 59, 381 (1987).

${ }^{43}$ J. P. Sethna, K. Dahmen, S. Kartha, J. A. Krumhansl, B. W. Roberts, and J. D. Shore, Phys. Rev. Lett. 70, 3347 (1993).

${ }^{44}$ M. A. Howson and B. L. Gallagher, Phys. Rep. 170, 265 (1988).

${ }^{45}$ P. A. Lee and T. V. Ramakrishnan, Rev. Mod. Phys. 57, 287 (1985).

${ }^{46}$ B. L. Al'tshuler, V. E. Kravtsov, and I. V. Lerner, Sov. Phys. JETP 64, 1352 (1986).

${ }^{47}$ N. F. Mott and E. A. Davis, Electronic Processes in NonCrystalline Materials (Clarendon, Oxford, 1971).

${ }^{48}$ C. C. Homes, T. Timusk, and X. Wu, Phys. Rev. Lett. 67, 2694 (1991).

${ }^{49}$ E. P. Wohlfahrt, in Magnetism Vol. 3, edited by G. T. Rado and H. Suhl (Academic, New York, 1963), p. 251.

${ }^{50}$ R. V. Chamberlin and F. Holtzberg, Phys. Rev. Lett. 67, 1606 (1991). 


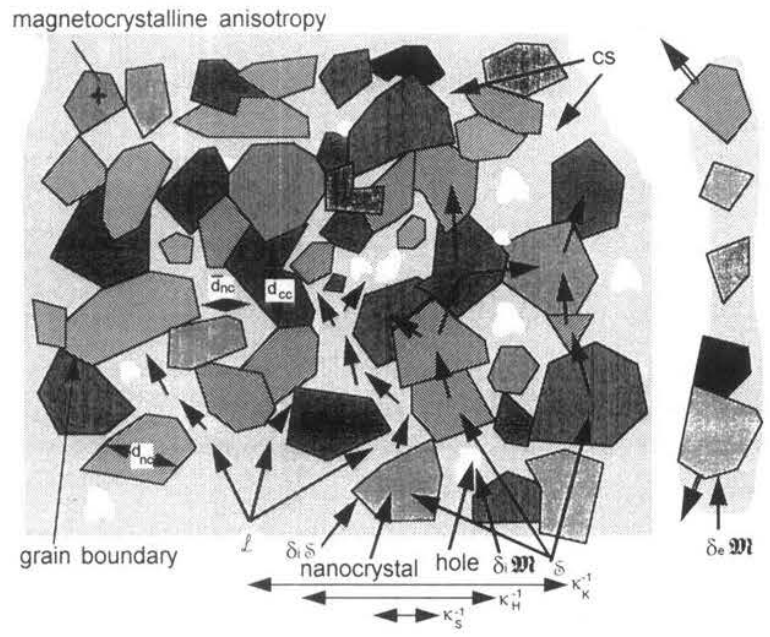

FIG. 1. Schematic illustration of a two-dimensional cross section of NS material characterized by three characteristic geometric lengths, $d_{\mathrm{NC}}, \overline{d_{\mathrm{NC}}}$, and $d_{\mathrm{CC}}$, and three characteristic magnetic lengths, $\kappa_{K}^{-1}, \kappa_{H}^{-1}$, and $\kappa_{S}^{-1}$. $\mathcal{S}$ (skeleton) and $\mathcal{L}$ (labyrinth) represent the global structural units of our model of NS material, with $d_{\mathrm{NC}}$ and $\overline{d_{\mathrm{NC}}}$ the linear scales of $\mathcal{S}$ and $\mathcal{L}$, respectively. $d_{\mathrm{CC}}$ represents the linear scale of crystallographic correlations of $\mathcal{S} . \partial_{i} \mathcal{M}$, and $\partial_{e} \mathcal{M}$ represent internal and external boundaries of the sample $\mathcal{M}$ with $\partial_{i} \mathcal{M}, \partial_{e} \mathcal{M}$ and $\partial_{i} \mathcal{\rho}$ representing the boundaries of holes (pores), the surface of the sample, and the surface of $\mathcal{S}$, respectively. 


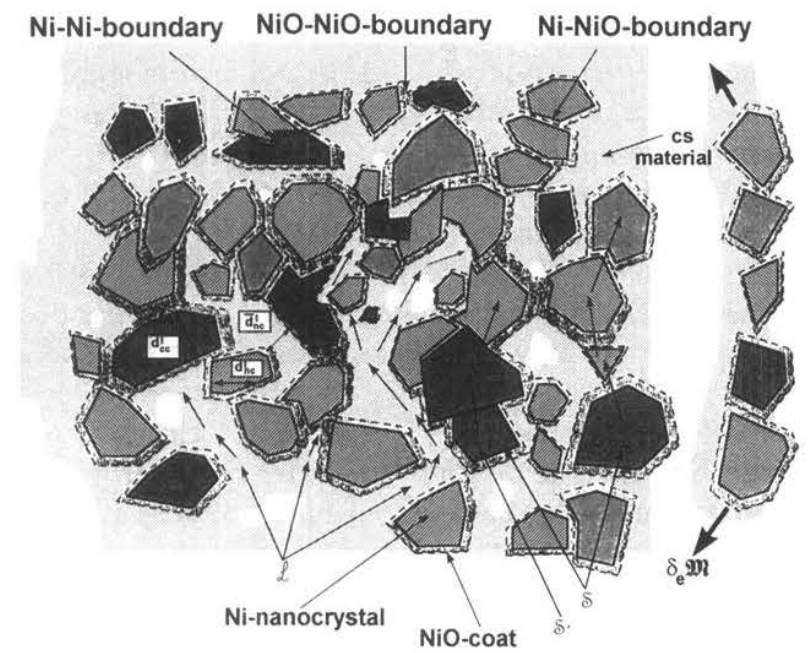

FIG. 5. Schematic illustration of a two-dimensional cross section of a NS material, whose building blocks are Ni nanocrystals surface coated with NiO. The coats of nanocrystals are indicated by dashed drawn lines. The labyrinth $\mathcal{L}$ contains CS material composed mainly of $\mathrm{NiO}$. The components of the mechanical skeleton $\mathcal{S}$ are joined by three types of grain boundaries $\mathrm{Ni}-\mathrm{Ni}, \mathrm{Ni}-\mathrm{NiO}$ and NiO-NiO. The components of the magnetic skeleton $\mathcal{f}^{\prime}$ are $\mathrm{Ni}$ nanocrystals joined by $\mathrm{Ni}-\mathrm{Ni}$ grain boundaries. The magnetic labyrinth $\mathcal{L}^{\prime}$ is defined via $\mathcal{L}^{\prime}+\mathcal{S}^{\prime}=\mathcal{M}^{\prime}$ but is not indicated. The characteristic lengths $d_{\mathrm{NC}}^{\prime}, \overline{d_{\mathrm{NC}}^{\prime}}$, and $d_{\mathrm{CC}}^{\prime}$ are defined with respect to $\mathcal{S}^{\prime}$ and $\mathcal{L}^{\prime}$ in analogy to Fig. 1. 